\title{
FINITE ELEMENT TRANSIENT ANALYSIS OF COMPOSITE AND SANDWICH PLATES BASED \\ ON A REFINED THEORY AND IMPLICIT TIME INTEGRATION SCHEMES
}

\author{
T. Kant, $\dagger$ J. H. VaraiYa and C. P. Arora \\ Department of Civil Engineering, Indian Institute of Technology, Powai, \\ Bombay 400 076, India
}

(Received 22 June 1989)

\begin{abstract}
An isoparametric finite element formulation based on a higher order displacement model for dynamic analysis of multilayer unsymmetric composite plates is presented. Newmark and Wilson- $\theta$ schemes are used for time integration of the discrete coupled second-order ordinary differential equation system of dynamic equilibrium and results are compared. A special mass diagonalization scheme is used which conserves total mass of the element and also includes the effects of rotary inertia terms. Effects of consistent/diagonal mass matrix, time step, and finite element mesh are investigated. Several numerical examples are presented and results are compared with those available in the literature.
\end{abstract}

\section{INTRODUCTION}

In recent years, due to the increased use of composite materials in the aerospace and automotive industries because of their superior mechanical properties such as high stiffness per unit weight, high strength per unit weight, and potentially low unit cost, a need has arisen for a basic understanding of their response to dynamic loading. The present study places emphasis on establishing the credibility of a higher order shear deformable (HOSD) finite element for transient analysis.

Laminated plate theories based on the Kirchhoff hypothesis were developed by Reissner and Stavsky [1] and Dong et al. [2]. The development of first-order shear deformable (FOSD) theory began with the work of Reissner [3] and Mindlin [4] for isotropic plates. For the analysis of thicker laminates, Mindlin's theory was extended to laminated anisotropic plates by Yang et al. [5] and by Whitney and Pagano [6]. Reismann and his colleagues [7-9] analysed a simply supported, rectangular, isotropic plate subjected to suddenly applied uniformly distributed load over a square area of the plate. Exact solution was obtained using classical three-dimensional elasticity theory and classical and improved plate theories. Rock and Hinton [10] presented transient finite element analysis of thick and thin isotropic plates using Reissner-Mindlin thick plate theory. Excellent agreement of the finite element solutions with the analytical solutions of Reismann and Lee [8] was obtained.

† To whom correspondence should be addressed.
Chow [11] employed the Laplace transform technique to investigate the dynamic response of orthotropic laminated plates. Wang et al. [12] applied the method of characteristics to unsymmetrical orthotropic laminated plates. Moon $[13,14]$ investigated the response of infinite laminated plates subjected to transverse impact loads at the centre of the plate. Sun and his colleagues [15-17] employed the classical method of separation of variables combined with Mindlin-Goodman [18] procedure for treating time dependent boundary conditions and/or dynamic loadings. All of these papers were confined to plates under cylindrical bending. Reddy $[19,20]$ presented closed form and finite element results for transient analysis of layered composite plates using MindlinReissner thick plate theory.

Theories based on displacement models which give rise to nonlinear distribution of inplane normal strains and transverse shear strains were developed by Lo et al. [21], Kant [22] and Phan and Reddy [23]. Lo et al. and Kant, in addition, included the effects of transverse normal strain and stress. Kant et al. [24] presented a $\mathrm{C}^{0}$ finite element formulation of a higher order theory. Pandya and Kant [25-28] extended the above formulation for generally orthotropic plates. All of these papers were confined to static analysis.

Recently, Mallikarjuna and Kan: [29] and Kant et al. [30] gave transient analysis of symmetrically/ unsymmetrically laminated composite plates based on a higher order displacement model using an explicit time integration scheme. The present paper specifically deals with the application of a higher order displacement model presented by one of the present authors [28] for investigating the transient response of isotropic and layered anisotropic plates. 
The finite element idealization is adopted using a nine-noded Lagrangian element. Implicit time integration schemes using consistent/diagonal mass matrices have been used for computing the transient response.

\section{THEORETICAL FORMULATION}

\subsection{Displacement model}

The present higher order shear deformation theory is based on the displacement model

$$
\begin{aligned}
u(x, y, z, t)= & u_{0}(x, y, t)+z \theta_{x}(x, y, t) \\
& +z^{2} u_{0}^{*}(x, y, t)+z^{3} \theta_{x}^{*}(x, y, t) \\
v(x, y, z, t)= & v_{0}(x, y, t)+z \theta_{y}(x, y, t) \\
& +z^{2} v_{0}^{*}(x, y, t)+z^{3} \theta_{y}^{*}(x, y, t) \\
w(x, y, z, t)= & w_{0}(x, y, t),
\end{aligned}
$$

where $t$ is time, $u_{0}, v_{0}$ and $w_{0}$ are the inplane and transverse displacements of a point $(x, y)$ on the mid-plane, respectively, and $\theta_{x}$ and $\theta_{y}$ are the rotations of normal to the mid-plane about the $y$ and $x$ axes, respectively. The parameters $u_{0}^{*}, v_{0}^{*}, \theta_{x}^{*}$ and $\theta_{y}^{*}$ are the corresponding higher order deformation terms in the Taylor series expansion and are also defined at the mid-plane. The parameters $u, v$ and $w$ are the displacement components in the $x, y$ and $z$ directions, respectively, of a generic point in the laminate space.

The generalized displacement vector at the midplane can thus be defined as

$$
\mathbf{d}=\left[u_{0}, v_{0}, w_{0}, \theta_{x}, \theta_{y}, u_{0}^{*}, v_{0}^{*}, \theta_{x}^{*}, \theta_{y}^{*}\right]^{T} .
$$

\subsection{Stress-strain relations in an orthotropic lamina}

In the present formulation, in conformity with the usual plate assumptions, the normal stress $\sigma_{3}$ can be assumed small enough to be neglected and the corresponding strain $\varepsilon_{3}$ is identically equal to zero. The generalized Hooke's law for a homogeneous orthotropic material may be written in a contracted notation as follows:

$$
\boldsymbol{\sigma}_{i}^{\prime}=\mathrm{C}_{i j} \boldsymbol{\varepsilon}_{j}^{\prime} \quad i, j=1,2,3,4,5
$$

or

$$
\sigma^{\prime}=\mathbf{C}^{\prime},
$$

in which $\sigma^{\prime}$ is the stress vector, $C$ is the composite material stiffness matrix and $\varepsilon^{\prime}$ is the engineering strain vector with reference to the principal material axes $(1,2,3)$.
The stress-strain relation for the $L$ th layer of the laminate can be expressed as

$$
\begin{aligned}
& {\left[\begin{array}{c}
\sigma_{1} \\
\sigma_{2} \\
\tau_{12} \\
\tau_{23} \\
\tau_{13}
\end{array}\right]^{L}=\left[\begin{array}{ccccc}
C_{11} & C_{12} & 0 & 0 & 0 \\
C_{12} & C_{22} & 0 & 0 & 0 \\
0 & 0 & C_{33} & 0 & 0 \\
0 & 0 & 0 & C_{44} & 0 \\
0 & 0 & 0 & 0 & C_{55}
\end{array}\right] } \\
& \times\left[\begin{array}{c}
\varepsilon_{1} \\
\varepsilon_{2} \\
\gamma_{12} \\
\gamma_{23} \\
\gamma_{13}
\end{array}\right]^{L},
\end{aligned}
$$

where

$$
\begin{aligned}
& C_{11}=E_{1} /\left(1-v_{12} v_{21}\right) \\
& C_{12}=v_{21} E_{1} /\left(1-v_{12} v_{21}\right) \\
& C_{22}=E_{2} /\left(1-v_{12} v_{21}\right) \\
& C_{33}=G_{12} \\
& C_{44}=G_{23} \\
& C_{55}=G_{13}
\end{aligned}
$$

and $E_{i}$ are the Young's moduli of elasticity in the $i$ direction, $v_{i j}$ are the Poisson's ratios that give strain in the $j$ direction due to stress in the $i$ direction and $G_{i j}$ are the shear moduli.

These relations are referred to the lamina principal axes $(1,2,3)$. The principal material axes of a lamina may not coincide with the reference axes of the laminated plate (Fig. 1). It is, therefore, necessary to transform the constitutive relations (5a) from the lamina principal axes to the reference axes of the laminate $(x, y, z)$. This is conveniently accomplished through a transformation as follows [31]:

$$
\begin{aligned}
& \boldsymbol{\sigma}^{\prime}=\mathbf{T} \boldsymbol{\sigma} \\
& \boldsymbol{\varepsilon}_{t s}^{\prime}=\mathbf{T} \boldsymbol{\varepsilon}_{t s},
\end{aligned}
$$

where $\sigma^{\prime}$ and $\varepsilon_{t s}^{\prime}$ are the vectors of stress and tensorial strain components, respectively, defined with reference to the lamina axes $(1,2,3), \sigma$ and $\varepsilon_{t s}$ are the vectors of stress and tensorial strain components, respectively, defined with reference to laminate axes $(x, y, z)$. The transformation vector $\mathbf{T}$ is given as

$$
\mathbf{T}=\left[\begin{array}{ccccc}
c^{2} & s^{2} & -2 s c & 0 & 0 \\
s^{2} & c^{2} & 2 s c & 0 & 0 \\
s c & -s c & c^{2}-s^{2} & 0 & 0 \\
0 & 0 & 0 & c & -s \\
0 & 0 & 0 & s & c
\end{array}\right],
$$




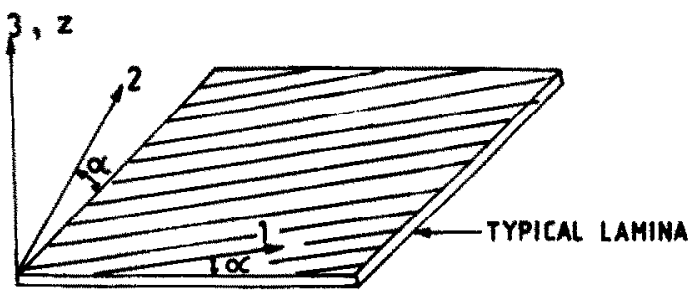

\section{$(1,2,3)$-LAMINA REFERENCE AXES}

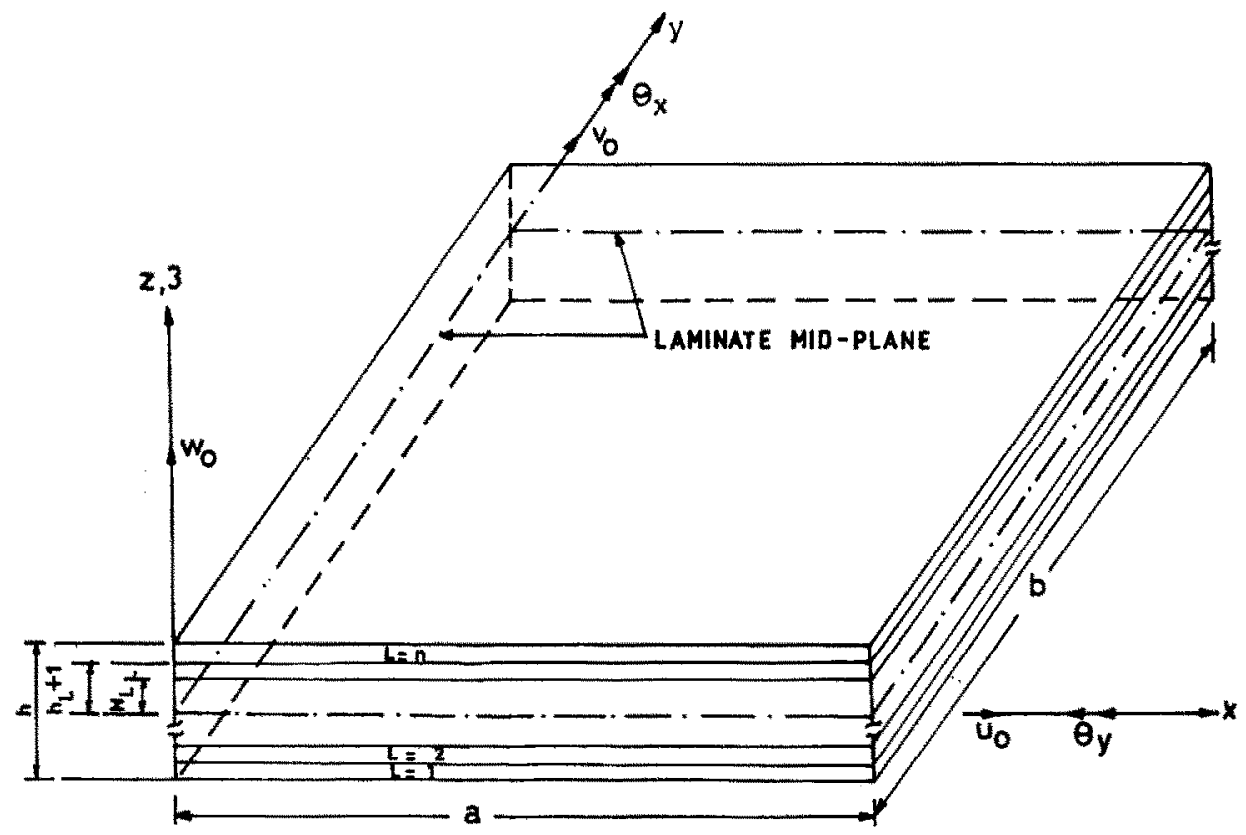

\section{$(x, y ; z)$ - LAMINATE REFERENCE axes}

Fig. 1. Laminate geometry with positive set of lamina/laminate reference axes, displacement components and fibre orientation.

in which $c=\cos \alpha$ and $s=\sin \alpha$, and $\alpha$ is the angle which the 1 and 2 directions make with the $x$ and $y$ axes, respectively.

The relationship between engineering and tensorial strain vectors is given by

$$
\boldsymbol{\varepsilon}^{\prime}=\mathbf{R} \boldsymbol{e}_{t s}^{\prime}
$$

and

$$
\boldsymbol{\varepsilon}=\mathbf{R}_{t s}
$$

or

$$
\boldsymbol{\varepsilon}_{t s}=\mathbf{R}^{-1} \mathbf{e}
$$

in which the $\mathbf{R}$ matrix is defined as

$$
\mathbf{R}=\left[\begin{array}{lllll}
1 & 0 & 0 & 0 & 0 \\
0 & 1 & 0 & 0 & 0 \\
0 & 0 & 2 & 0 & 0 \\
0 & 0 & 0 & 2 & 0 \\
0 & 0 & 0 & 0 & 2
\end{array}\right]
$$

The stress-strain constitutive relations with reference to the laminate axes are shown in the following expression by making use of relations (4), (6) and (9):

$$
\sigma=\mathbf{T}^{-1} \mathbf{C R T R}^{-1} \boldsymbol{c}
$$

It can easily be proved that

$$
\mathbf{R T R}^{-1}=\mathbf{T}^{-1 t} .
$$

Thus, the relation (11) can be rewritten as

$$
\sigma=\mathbf{Q} \boldsymbol{\varepsilon}
$$

in which

$$
\mathbf{Q}=\mathbf{T}^{-1} \mathbf{C} \mathbf{T}^{-1 T}
$$

(10) The superscripts $T$ and -1 represent the transpose and inverse of a matrix, respectively, and the inverse of the transformation matrix is obtained by replacing $\alpha$ by $-\alpha$ in eqn (7). After triple product of the $\mathbf{T}^{-1}$, 
$\mathbf{C}$ and $\mathbf{T}^{-1 r}$ matrices, the $\mathbf{Q}$ matrix coefficients are obtained and are defined as

$$
\begin{aligned}
& Q_{11}=C_{11} c^{4}+2\left(C_{12}+4 C_{33}\right) s^{2} c^{2}+C_{22} s^{4} \\
& Q_{12}=C_{12}\left(c^{4}+s^{4}\right)+\left(C_{11}+C_{22}+4 C_{33}\right) s^{2} c^{2} \\
& Q_{13}=\left(C_{11}-C_{12}-2 C_{33}\right) c^{3} s+\left(C_{12}-C_{22}+2 C_{33}\right) s^{3} c \\
& Q_{22}=C_{11} s^{4}+C_{22} c^{4}+\left(2 C_{11}+4 C_{33}\right) s^{2} c^{2} \\
& Q_{23}=\left(C_{11}-C_{12}-2 C_{33}\right) s^{3} c+\left(C_{12}-C_{22}+2 C_{33}\right) c^{3} s \\
& Q_{33}=\left(C_{11}-2 C_{12}+2 C_{22}-2 C_{33}\right) c^{2} s^{2}+C_{33}\left(c^{4}+s^{4}\right) \\
& Q_{44}=C_{44} c^{2}+C_{55} s^{2} \\
& Q_{45}=\left(C_{55}-C_{44}\right) c s \\
& Q_{55}=C_{44} s^{2}+C_{55} c^{2} \\
& Q_{i j}=Q_{j i}, \quad i, j=1,2,3,4,5 .
\end{aligned}
$$

Thus, the strain relations for an orthotropic lamina with reference to the laminate axes are defined as

$$
\begin{aligned}
& {\left[\begin{array}{c}
\sigma_{x} \\
\sigma_{y} \\
\tau_{x y} \\
\tau_{y z} \\
\tau_{x z}
\end{array}\right]^{L}=\left[\begin{array}{lllll}
Q_{11} & Q_{12} & Q_{13} & 0 & 0 \\
& Q_{22} & Q_{23} & 0 & 0 \\
& & Q_{33} & 0 & 0 \\
& & Q_{44} & Q_{45} \\
\text { Symm. } & & & Q_{55}
\end{array}\right]^{L} } \\
& \times\left[\begin{array}{c}
\varepsilon_{x} \\
\varepsilon_{y} \\
\gamma_{x y} \\
\gamma_{y z} \\
\gamma_{x z}
\end{array}\right]^{L}
\end{aligned}
$$

\subsection{Strain expressions}

The strains associated with displacements in eqn (1) are as follows:

$$
\begin{aligned}
\varepsilon_{x} & =\varepsilon_{x 0}+z \kappa_{x}+z^{2} \varepsilon_{x 0}^{*}+z^{3} \kappa_{x}^{*} \\
\varepsilon_{y} & =\varepsilon_{y 0}+z \kappa_{y}+z^{2} \varepsilon_{y 0}^{*}+z^{3} \kappa_{y}^{*} \\
\varepsilon_{z} & =0 \\
\gamma_{x y} & =\varepsilon_{x y 0}+z \kappa_{x y}+z^{2} \varepsilon_{x y 0}^{*}+z^{3} \kappa_{x y}^{*} \\
\gamma_{y z} & =\phi_{y}+z \psi_{y}+z^{2} \phi_{y}^{*} \\
\gamma_{x z} & =\phi_{x}+z \psi_{x}+z^{2} \phi_{x}^{*},
\end{aligned}
$$

where

$$
\begin{aligned}
& \varepsilon_{0}=\left[\varepsilon_{x 0}, \varepsilon_{y 0}, \varepsilon_{x, 0}\right]^{T}=\left[\frac{\partial u_{0}}{\partial x}, \frac{\partial v_{0}}{\partial y}, \frac{\partial u_{0}}{\partial y}+\frac{\partial v_{0}}{\partial x}\right]^{T} \\
& \boldsymbol{\varepsilon}_{0}^{*}=\left[\varepsilon_{x 0}^{*}, \varepsilon_{y 0}^{*}, \varepsilon_{x y 0}^{*}\right]^{T}=\left[\frac{\partial u_{0}^{*}}{\partial x}, \frac{\partial v_{0}^{*}}{\partial y}, \frac{\partial u_{0}^{*}}{\partial y}+\frac{\partial v_{0}^{*}}{\partial x}\right]^{T} \\
& \boldsymbol{\kappa}=\left[\kappa_{r}, \kappa_{y}, \kappa_{x y}\right]^{T}=\left[\frac{\partial \theta_{x}}{\partial x}, \frac{\partial \theta_{y}}{\partial y}, \frac{\partial \theta_{x}}{\partial y}+\frac{\partial \theta_{y}}{\partial x}\right]^{T}
\end{aligned}
$$

$$
\begin{aligned}
\boldsymbol{\kappa}^{*} & =\left[\boldsymbol{\kappa}_{x}^{*}, \boldsymbol{\kappa}_{y}^{*}, \kappa_{x y}^{*}\right]^{T}=\left[\frac{\partial \theta_{x}^{*}}{\partial x}, \frac{\partial \theta_{y}^{*}}{\partial y}, \frac{\partial \theta_{x}^{*}}{\partial y}+\frac{\partial \theta_{y}^{*}}{\partial x}\right]^{T} \\
\boldsymbol{\phi} & =\left[\boldsymbol{\phi}_{x}, \phi_{y}\right]^{T}=\left[\theta_{x}+\frac{\partial w_{0}}{\partial x}, \theta_{y}+\frac{\partial w_{0}}{\partial y}\right]^{T} \\
\boldsymbol{\phi}^{*} & =\left[\phi_{x}^{*}, \phi_{y}^{*}\right]^{T}=\left[3 \theta_{x}^{*}, 3 \theta_{y}^{*}\right]^{T} \\
\boldsymbol{\psi} & =\left[\psi_{x}, \psi_{y}\right]^{T}=\left[2 u_{0}^{*}, 2 v_{0}^{*}\right]^{T}
\end{aligned}
$$

and $T$ represents transpose of an array.

The generalized strain vector $\bar{\varepsilon}$ corresponding to the middle surface may be written as

$$
\begin{array}{r}
\bar{\varepsilon}=\left[\varepsilon_{x 0}, \varepsilon_{y 0}, \varepsilon_{x y 0}, \varepsilon_{x 0}^{*}, \varepsilon_{y 0}^{*}, \varepsilon_{x y 0}^{*}, \kappa_{x}, \kappa_{y}, \kappa_{x y}, \kappa_{x}^{*},\right. \\
\left.\kappa_{y}^{*}, \kappa_{x y}^{*}, \phi_{x}, \phi_{y}, \psi_{x}, \psi_{y}, \phi_{x}^{*}, \phi_{y}^{*}\right]^{T} .
\end{array}
$$

\subsection{Equations of motion}

The Hamilton variational principle is used here to derive the laminate equations of motion. The mathematical statement of the Hamilton principle in the absence of damping can be written as

$$
\int_{t_{1}}^{t_{2}} \delta(\Pi-E) \mathrm{d} t=0
$$

where $\Pi$ and $E$ are the total potential energy and the kinetic energy, respectively.

The potential energy $\Pi$ of the plate with volume $V$ and surface area $A$ can be written as

$$
\Pi=U-W
$$

or

$$
\Pi=1 / 2 \int_{v} \varepsilon^{T} \boldsymbol{\sigma} \mathrm{d} V-\int_{v} \mathbf{u}^{T} \mathbf{P} \mathrm{d} A,
$$

where $U$ is the strain energy of the plate; $W$ represents the work done by externally applied forces; $\mathbf{P}$ is the vector of force intensities corresponding to laminate direction $(x, y, z)$; and $\mathbf{u}=[u, v, w]^{T}$ is the displacement of any generic point $(x, y, z)$ in space. The expressions for the strain components given by eqn (16) are substituted in the energy expression (19). An explicit integration through the laminate thickness is then carried out to obtain the following expression:

$$
\Pi=1 / 2 \int_{A} \bar{\varepsilon}^{T} \bar{\sigma} \mathrm{d} A-\int_{A} \mathrm{~d}^{T} \mathbf{F} \mathrm{d} A,
$$

in which $\mathbf{F}$ is the vector of load per unit area corresponding to the direction of generalized displacement vector $\mathbf{d}$ and the new vector $\bar{\sigma}$ is defined in the following manner:

$$
\begin{gathered}
\overline{\boldsymbol{\sigma}}=\left[N_{x}, N_{y}, N_{x y}, N_{x}^{*}, N_{y}^{*}, N_{x y}^{*}, M_{x}, M_{y}, M_{x y},\right. \\
\left.M_{x}^{*}, M_{y}^{*}, M_{x y}^{*}, Q_{x}, Q_{y}, S_{x}, S_{y}, Q_{x}^{*}, Q_{y}^{*}\right]^{T}
\end{gathered}
$$


Equation (20) defines the two-dimensional form of the potential energy in which the relationship between $\bar{\sigma}$ and $\bar{\varepsilon}$ will now define the two-dimensional laminate constitutive relations. On integration of stresses over the laminate thickness, these relations are obtained. The following 18 stress resultants for the $N L$-layered laminate are thus derived:

$$
\begin{gathered}
\left(\begin{array}{c}
N_{x}, N_{y}, N_{x y} \\
N_{x}^{*}, N_{y}^{*}, N_{x y}^{*}
\end{array}\right)=\sum_{L=1}^{N L} \int_{h_{L}}^{h_{L+1}}\left(\begin{array}{c}
1 \\
z^{2}
\end{array}\right)\left[\sigma_{x} \sigma_{y} \tau_{x y}\right] \mathrm{d} z \\
\left(\begin{array}{c}
M_{x}, M_{y}, M_{x y} \\
M_{x}^{*}, M_{y}^{*}, M_{x y}^{*}
\end{array}\right)=\sum_{L=1}^{N L} \int_{h_{L}}^{h_{L+1}}\left(\begin{array}{c}
z \\
z^{3}
\end{array}\right)\left[\begin{array}{lll}
\sigma_{x} & \sigma_{y} & \tau_{x y}
\end{array}\right] \mathrm{d} z \\
\left(\begin{array}{l}
Q_{x}, S_{x}, Q_{x}^{*} \\
Q_{y}, S_{y}, Q_{y}^{*}
\end{array}\right)=\sum_{L=1}^{N L} \int_{h_{L}}^{h_{L+1}}\left(\begin{array}{c}
\tau_{x z} \\
\tau_{y z}
\end{array}\right)\left[\begin{array}{lll}
1 & z & z^{2}
\end{array}\right] \mathrm{d} z .
\end{gathered}
$$

The stresses in eqns (22) are replaced with midplane strains and then the following relations between mid-plane stress resultants and strains are obtained:

$$
\begin{aligned}
{\left[\begin{array}{c}
\mathbf{N} \\
\mathbf{N}^{*} \\
\mathbf{M} \\
\mathbf{M}^{*}
\end{array}\right]=} & \sum_{L=1}^{N L}\left[\begin{array}{llll}
\mathbf{Q}_{i j} H_{1} & \mathbf{Q}_{i j} H_{3} & \mathbf{Q}_{i j} H_{2} & \mathbf{Q}_{i j} H_{4} \\
& \mathbf{Q}_{i j} H_{5} & \mathbf{Q}_{i j} H_{4} & \mathbf{Q}_{i j} H_{6} \\
& & \mathbf{Q}_{i j} H_{3} & \mathbf{Q}_{i j} H_{5} \\
& & \mathbf{Q}_{i j} H_{7}
\end{array}\right) \\
& \times\left(\begin{array}{c}
\boldsymbol{\varepsilon}_{0}^{*} \\
\boldsymbol{\varepsilon}_{0}^{*} \\
\boldsymbol{\kappa} \\
\boldsymbol{\kappa}^{*}
\end{array}\right] \\
\left(\begin{array}{c}
\mathbf{Q} \\
\mathbf{S} \\
\mathbf{Q}^{*}
\end{array}\right)= & \sum_{L=1}^{N L}\left(\begin{array}{lll}
\mathbf{Q}_{m l} H_{1} & \mathbf{Q}_{m l} H_{2} & \mathbf{Q}_{m l} H_{3} \\
& \mathbf{Q}_{m l} H_{3} & \mathbf{Q}_{m l} H_{4} \\
& & \mathbf{Q}_{m l} H_{5}
\end{array}\right)\left(\begin{array}{c}
\boldsymbol{\phi} \\
\boldsymbol{\psi} \\
\boldsymbol{\phi}^{*}
\end{array}\right),
\end{aligned}
$$

where $i, j=1,2,3$ and $l, m=4,5$.

The terms $\varepsilon_{0}, \varepsilon_{0}^{*}, \kappa, \kappa^{*}, \phi, \phi^{*}$ and $\psi$ are defined in eqn (16), and

$$
\begin{aligned}
\mathbf{M} & =\left[M_{x}, M_{y}, M_{x y}\right]^{T} \\
\mathbf{M}^{*} & =\left[M_{x}^{*}, M_{y}^{*}, M_{x y}^{*}\right]^{T} \\
\mathbf{N} & =\left[N_{x}, N_{y}, N_{x y}\right]^{T} \\
\mathbf{N}^{*} & =\left[N_{x}^{*}, N_{y}^{*}, N_{x y}^{*}\right]^{T} \\
\mathbf{Q} & =\left[Q_{x}, Q_{y}\right]^{T} \\
\mathbf{Q}^{*} & =\left[Q_{x}^{*}, Q_{y}^{*}\right]^{T} \\
\mathbf{S} & =\left[S_{x}, S_{y}\right]^{T} .
\end{aligned}
$$

In all of the above relations $N L$ is the number of layers and

$$
H_{i}=\left(h_{L+1}^{i}-h_{L}^{i}\right) / i, \quad i=1,2,3,4,5,6,7 .
$$

The above relations can be concisely expressed as

$$
\bar{\sigma}=\mathbf{D} \bar{\varepsilon}
$$

or

$$
\left[\begin{array}{c}
\mathbf{N} \\
\mathbf{N}^{*} \\
\hline \mathbf{M} \\
\mathbf{M}^{*} \\
\hline \mathbf{Q} \\
\mathbf{S} \\
\mathbf{Q}^{*}
\end{array}\right]=\left[\begin{array}{c|c|c}
\mathbf{D}_{m} & \mathbf{D}_{c} & 0 \\
\hdashline 0 & 0 & D_{s} \\
\hdashline & \mathbf{D}_{b} & 0 \\
\hdashline \\
\frac{\boldsymbol{\alpha}}{\boldsymbol{\psi}} \\
\boldsymbol{\theta}^{*}
\end{array}\right]
$$

Thus, with the assumed displacement model, the various rigidity matrices derived are

$\mathbf{D}_{m}$ : membrane; $\mathbf{D}_{c}$ : membrane-flexure coupling

$\mathbf{D}_{b}$ : flexure; $\mathbf{D}_{s}$ : shear.

\subsection{Interlaminar stresses}

The interlaminar transverse stresses $\left(\tau_{x z}, \tau_{y z}, \sigma_{z}\right)$ cannot be accurately estimated by eqn (15). This is mainly due to the fact that the constitutive laws are discontinuous across the laminae interfaces whereas interlaminar stresses have to maintain continuity across the interfaces. For these reasons, the interlaminar stresses between the layers ' $L$ ' and ' $L+1$ ' are obtained by integrating the following three equilibrium equations of three-dimensional elasticity for each layer over the lamina thickness and summing over layers 1 to ' $L$ ':

$$
\begin{aligned}
& \frac{\partial \sigma_{x}}{\partial x}+\frac{\partial \tau_{x y}}{\partial y}+\frac{\partial \tau_{x z}}{\partial z}=0 \\
& \frac{\partial \tau_{x y}}{\partial x}+\frac{\partial \sigma_{y}}{\partial y}+\frac{\partial \tau_{y z}}{\partial z}=0 \\
& \frac{\partial \tau_{x z}}{\partial x}+\frac{\partial \tau_{y z}}{\partial y}+\frac{\partial \sigma_{z}}{\partial z}=0 .
\end{aligned}
$$

The above equilibrium equations without body forces are rewritten in the following form for computation:

$$
\begin{aligned}
& \left.\tau_{x z}^{L}\right|_{z=h_{L+1}}=-\sum_{i=1}^{L} \int_{h_{i}}^{h_{i+1}}\left(\frac{\partial \sigma_{x}^{i}}{\partial x}+\frac{\partial \tau_{x y}^{i}}{\partial y}\right) \mathrm{d} z \\
& \left.\tau_{y z}^{L}\right|_{z=h_{L+1}}=-\sum_{i=1}^{L} \int_{h_{i}}^{h_{i+1}}\left(\frac{\partial \sigma_{y}^{i}}{\partial y}+\frac{\partial \tau_{x y}^{i}}{\partial x}\right) \mathrm{d} z \\
& \left.\sigma_{z}^{L}\right|_{z=h_{L+1}}=-\sum_{i=1}^{L} \int_{h_{i}}^{h_{i+1}}\left(\frac{\partial \tau_{x z}^{i}}{\partial x}+\frac{\partial \tau_{y z}^{i}}{\partial y}\right) \mathrm{d} z .
\end{aligned}
$$




\subsection{Kinetic energy}

The kinetic energy of the body is given by

$$
E=1 / 2 \int_{v} \dot{\mathbf{u}}^{T} \rho \dot{\mathbf{u}} \mathrm{d} v
$$

where $\dot{\mathbf{u}}$ is the velocity vector of any generic point $(x, y, z)$ in space and $\rho$ is the mass density of the material. The expression for $\dot{u}$ is substituted in eqn (30) using eqn (1) and, on carrying out explicit integration through the thickness of the laminate, this relation can be reduced into a two-dimensional form as

$$
E=1 / 2 \int_{A} \mathbf{d}^{T-} \overline{\mathbf{m}} \mathbf{d} \mathrm{d} A,
$$

where the inertia matrix $\vec{m}$ is given as

$$
\tilde{\mathbf{m}}=\left[\begin{array}{ccccccccc}
I_{0} & 0 & 0 & I_{1} & 0 & I_{2} & 0 & I_{3} & 0 \\
0 & I_{0} & 0 & 0 & I_{1} & 0 & I_{2} & 0 & I_{3} \\
0 & 0 & I_{0} & 0 & 0 & 0 & 0 & 0 & 0 \\
I_{1} & 0 & 0 & I_{2} & 0 & I_{3} & 0 & I_{4} & 0 \\
0 & I_{1} & 0 & 0 & I_{2} & 0 & I_{3} & 0 & I_{4} \\
I_{2} & 0 & 0 & I_{3} & 0 & I_{4} & 0 & I_{5} & 0 \\
0 & I_{2} & 0 & 0 & I_{3} & 0 & I_{4} & 0 & I_{5} \\
I_{3} & 0 & 0 & I_{4} & 0 & I_{5} & 0 & I_{6} & 0 \\
0 & I_{3} & 0 & 0 & I_{4} & 0 & I_{5} & 0 & I_{6}
\end{array}\right],
$$

in which

$$
I_{i}=\sum_{L=1}^{N L} \int_{h_{L}}^{h_{L+1}} z^{i} \rho^{L} \mathrm{~d} z, \quad i=0,6,
$$

and $\rho^{L}$ is the material density of the $L$ th layer.

The substitution of eqns (20), (26) and (30) into the mathematical statement of the Hamilton principle gives

$$
\begin{aligned}
\delta \int_{I^{\prime}}^{t_{2}} & {\left[1 / 2 \int_{A} \overline{\boldsymbol{\varepsilon}}^{T} \mathbf{D} \bar{\varepsilon} \mathrm{d} A\right.} \\
& \left.-\int_{A} \mathbf{d}^{T} \mathbf{F} \mathrm{d} A-1 / 2 \int_{A} \mathbf{d}^{T} \overline{\mathbf{m}} \mathbf{d} A\right] \mathrm{d} t=0 .
\end{aligned}
$$

The application of this principle furnishes the equations of motion of any given system.

\section{FINITE ELEMENT DISCRETIZATION}

\subsection{Discretization in space}

In standard finite element technique, the total solution domain is discretized into ' $N E$ ' subdomains (elements), such that

$$
\begin{aligned}
& \Pi(\mathbf{d})=\sum_{e=1}^{N E} \Pi_{e}(\mathbf{d}) \\
& E(\mathbf{d})=\sum_{e=1}^{N E} E_{e}(\mathbf{d}),
\end{aligned}
$$

where $\Pi$ and $\Pi_{e}$ are the total potential energies of the system and the element, respectively, and $E$ and $E_{e}$ are the kinetic energies of the system and element, respectively. The basic discretization process is carried over an element only and then the resulting equations are summed over the entire domain.

\subsection{Displacement function}

The continuum displacement vector within the element is then discretized such that

$$
\mathbf{d}(t)=\sum_{i=1}^{N N} \mathbf{N}_{i}(x, y) \mathbf{d}_{i}(t),
$$

where $\mathbf{N}_{i}$ is the interpolation function corresponding to node $i, N N$ is the number of nodes in the element and $\mathbf{d}_{i}$ is the vector of nodal degrees of freedom corresponding to node $i$, such that

$$
\mathbf{d}_{i}=\left[u_{0 i}, v_{0 i}, w_{0 i}, \theta_{x i}, \theta_{y i}, u_{0 i}^{*}, v_{0 i}^{*}, \theta_{x i}^{*}, \theta_{y i}^{*}\right]^{T}
$$

Equation (33) can be written as

$$
\mathbf{d}(t)=\mathbf{N a} \mathbf{a}_{e}(t)
$$

where $\mathbf{N}$ is the shape function matrix given by

$$
\mathbf{N}=\left[\mathbf{N}_{1}, \mathbf{N}_{2} \ldots \mathbf{N}_{N N}\right]
$$

and $\mathbf{a}_{e}$ is the element displacement vector which is written as

$$
\mathbf{a}_{e}=\left[\mathbf{d}_{1}^{T}, \mathbf{d}_{2}^{T} \ldots \mathbf{d}_{N N}^{T}\right]^{T} .
$$

Knowing the generalized displacement vector $\mathbf{d}$ at all points within the element, the generalized strain at any point, given by eqn (16), can be expressed in matrix form as follows:

$$
\bar{\varepsilon}=\sum_{i=1}^{N N} \mathbf{B}_{i} \mathbf{d}_{i}
$$

Equation (38) is also written in an alternative form as

$$
\overline{\mathbf{\varepsilon}}=\mathbf{B} \mathbf{a}_{e}
$$

in which

$$
\mathbf{B}=\left[\mathbf{B}_{1}, \mathbf{B}_{2} \ldots \mathbf{B}_{N N}\right]
$$


The non-zero elements of the strain-displacement matrix $\mathrm{B}_{i}$, which has a dimension of $(18 \times 9)$, are given below.

$$
\begin{aligned}
B_{1.1}=B_{3,2}=B_{4,6}=B_{6,7}=B_{7,4} & =B_{9,5}=B_{10,8} \\
& =B_{12,9}=B_{19,3}=\frac{\partial N_{i}}{\partial x} \\
B_{2,2}=B_{3,1}=B_{5,7}=B_{6,6}=B_{8,5} & =B_{9,4}=B_{11,9} \\
& =B_{12,8}=B_{14,3}=\frac{\partial N_{i}}{\partial y} \\
B_{13,4}=B_{14,5} & =N_{i} \\
B_{15,6}= & B_{16,7}=2 N_{i} \\
B_{17,8}= & B_{18,9}=3 N_{i} .
\end{aligned}
$$

\subsection{Discrete equations of motion}

The potential energy for an element ' $e$ ' can be expressed in terms of the internal strain energy $U_{e}$ and the external work done $W_{e}$, such that

$$
\Pi_{e}=U_{e}-W_{e} .
$$

For an element, the internal strain energy [ref. eqns (20) and (31)] can be written as

$$
U_{e}=1 / 2 \mathbf{a}_{e}^{T} K_{e} \mathbf{a}_{e}
$$

and the first variation of internal strain energy can be written in matrix form as

$$
\partial U_{e}=\delta \mathbf{a}_{e}^{T} \mathbf{K}_{e} \mathbf{a}_{e},
$$

where

$$
\mathbf{K}_{e}=\int_{A} \mathbf{B}^{T} \mathbf{D B} \mathrm{d} A
$$

is the element stiffness matrix.

The first variation of work done on the laminate by the external loads, $\delta W$, for an element [ref. eqns (20) and (31)] can be written in matrix form as

$$
\delta W_{e}=\delta \mathbf{a}_{e}^{T} \mathbf{f}_{e}(t),
$$

in which the element equivalent nodal load vector

$$
\mathbf{f}_{e}(t)=\int_{1} \mathbf{N}^{T} \mathbf{F} \mathrm{d} A
$$

and

$$
\mathrm{F}=[0,0, q, 0,0,0,0,0,0]^{T},
$$

where $q$ is the intensity of the applied transverse load.
The first variation of kinetic energy, $\delta E$, for an element [ref. eqn (30)] can be written in matrix form as

$$
\delta E_{e}=-\delta \mathfrak{a}_{e}^{T}\left[\int_{A} \mathbf{N}^{T} \mathbf{m} \mathbf{N} \mathrm{d} A\right] \ddot{a}_{e}=-\delta \mathfrak{a}_{e}^{T} \mathbf{M}_{e} \ddot{\mathbf{a}}_{e},
$$

where

$$
\mathbf{M}_{e}=\int_{A} \mathbf{N}^{T} \overline{\mathbf{m}} \mathbf{N} \mathrm{d} A
$$

is the element consistent mass matrix, $\ddot{a}$ is the vector of element nodal acceleration, $\mathbf{N}$ is the shape function matrix and $\overline{\mathbf{m}}$ is the inertia matrix.

When expressions for $\delta U, \delta W$ and $\delta E$ from eqns (43), (44) and (45) are substituted in eqn (18) or (31), we obtain

$$
\int_{t_{1}}^{t_{2}} \delta \mathbf{a}_{e}^{T}\left[M_{e} \ddot{\mathbf{a}}_{e}+\mathbf{K}_{e} \mathbf{a}_{e}-\mathbf{f}_{e}(t)\right] \mathrm{d} t=0
$$

Since this relation is valid for every virtual displacement $\delta \mathbf{a}_{e}$, one obtains

$$
\mathbf{M}_{e} \ddot{\mathbf{z}}_{e}+\mathbf{K}_{e} \mathbf{a}_{e}=\mathbf{f}_{e}(t),
$$

which is the finite element equation of motion of one element of the laminate. The element equations are then assembled as per eqn (32) to yield the global equations of motion for the entire laminate, i.e.

$$
\mathbf{M a ̈}+\mathbf{K a}=\mathbf{f}(t),
$$

in which and a are the global vectors of unknown acceleration and displacement, respectively. The global stiffness matrix $\mathbf{K}$, the global mass matrix $\mathbf{M}$ and the global nodal force vector $\mathrm{f}(t)$ are computed in the usual manner as follows:

$$
\begin{gathered}
\mathbf{M}=\sum_{e=1}^{N E} \mathbf{M}_{e} \\
\mathbf{K}=\sum_{e=1}^{N E} \mathbf{K}_{e} \\
\mathbf{f}(t)=\sum_{e=1}^{N E} \mathbf{f}_{e}(t) .
\end{gathered}
$$

\subsection{Element stiffness matrix}

The computation of element stiffness matrix $\mathbf{K}^{\boldsymbol{e}}$ from eqn (43) is economized by explicit multiplication of matrices $\mathbf{B}_{i}, \mathbf{D}$ and $\mathbf{B}_{j}$ matrices instead of carrying out the full matrix multiplication of the triple product. In addition, due to symmetry of the stiffness matrix, only the blocks $\mathbf{K}_{i j}$ laying on one side of the main diagonal are formed. The integral is evaluated by selective integration technique with $3 \times 3$ and 
$2 \times 2$ Gauss quadrature rules for membrane-flexure and shear parts, respectively, as follows:

$$
\begin{aligned}
& \mathbf{K}_{i j}^{e}=\int_{1}^{+1} \int_{-1}^{+1} \mathbf{B}_{i}^{T} \mathbf{D} \mathbf{B}_{j}|\mathbf{J}| \mathrm{d} \xi \mathrm{d} \eta \\
& \mathbf{K}_{i j}^{e}=\sum_{a=1}^{g} \sum_{b=1}^{g} W_{a} W_{b}|\mathbf{J}| \mathbf{B}_{i}^{T} \mathbf{D B}_{j},
\end{aligned}
$$

where $W_{u}$ and $W_{b}$ are the weighting coefficients, $g$ is the number of numerical quadrature points in each of the two directions $(x, y)$, and $|\mathbf{J}|$ is the determinant of the standard Jacobian matrix. The subscripts $i$ and $j$ vary from one node to a number of nodes per element. The matrices $B_{i}$ and $D$ are given by eqns (40) and (26), respectively.

\subsection{Element mass matrix}

The computation of the consistent mass matrix $\mathbf{M}^{e}$ from eqn (45) is evaluated using the $3 \times 3$ Gauss quadrature rule as follows:

$$
\begin{aligned}
& \mathbf{M}_{i j}^{e}=\int_{-1}^{+1} \int_{-1}^{+1} \mathbf{N}_{i}^{T} \overline{\mathbf{m}} \mathbf{N}_{j}|\mathbf{J}| \mathrm{d} \xi \mathrm{d} \eta \\
& \mathbf{M}_{i j}^{e}=\sum_{a=1}^{g} \sum_{b=1}^{g} W_{a} W_{b}|\mathbf{J}| \mathbf{N}_{i}^{T} \overline{\mathbf{m}} \mathbf{N}_{j},
\end{aligned}
$$

where the subscripts $i$ and $j$ vary from one node to number of nodes per element and $\mathbf{N}_{i}$ is the interpolation function corresponding to node $i$.

Several alternatives for obtaining the diagonal mass matrix of isoparametric elements were investigated by Hinton et al. [32]. The most efficient scheme found to date and which is used herein can be summarized as follows [33].

1. Compute only the diagonal coefficients of the consistent mass matrix, $M_{i i}^{e}$.

2. Compute the total mass of the element, $M^{e}=\int_{v} \rho \mathrm{d} v$.

3. Compute a quantity $S$ by adding only the coefficients $\boldsymbol{M}_{i i}^{e}$ associated with translation degrees of freedom in one direction.

4. Scale all the diagonal coefficients $\boldsymbol{M}_{i i}^{e}$ by multiplying them with the ratio $M^{e} / S$, thus preserving the total mass and rotary inertia of the element.

\subsection{Element load vector}

The consistent load vector $\mathbf{f}_{i}$ due to body force

$$
q=q_{0}+P_{m n} \sin \frac{m \pi x}{a} \sin \frac{n \pi y}{b}
$$

is given by

$$
\mathbf{f}_{i}(t)=\int_{A} \mathbf{N}_{i}^{T} \mathbf{F} \mathrm{d} A .
$$

The integral of eqn (55) is evaluated numerically using the Gauss quadrature rule as follows:

$$
\begin{array}{r}
\mathbf{f}_{i}=\sum_{u=1}^{g} \sum_{b=1}^{g} W_{a} W_{b}|\mathbf{J}| \mathbf{N}_{i}^{T}[0,0,1,0,0,0,0,0,0]^{T} \\
\times\left(q_{0}+P_{m n} \sin \frac{m \pi x}{a} \sin \frac{n \pi y}{b}\right),
\end{array}
$$

where $x$ and $y$ are the Gauss point coordinates, and $m$ and $n$ are the usual harmonic numbers.

To complete the discretization, we must now approximate the time derivatives appearing in eqn (48). Here we use the Newmark or Wilson- $\theta$ direct integration methods $[34,35]$.

\subsection{Newmark direct time integration method}

In this scheme the vectors $\mathbf{a}$ and $\mathbf{a}$ at the end of a time step $\Delta t$ are expressed in the form

$$
\begin{aligned}
& \dot{\mathbf{a}}^{n+1}=\dot{\mathbf{a}}^{n}+\Delta t\left[(1-\delta) \ddot{\mathbf{a}}^{n}+\delta \ddot{\mathbf{a}}^{n+1}\right] \\
& \mathbf{a}^{n+1}=\mathbf{a}^{n}+\Delta t \dot{\mathbf{a}}^{n}+(\Delta t)^{2}\left[(1 / 2-\alpha) \ddot{\mathbf{a}}^{n}+\alpha \ddot{\mathbf{a}}^{n+1}\right],
\end{aligned}
$$

where $\alpha$ and $\delta$ are parameters that control the accuracy and stability of the scheme and the superscript $n$ indicates that the solution is evaluated at the $n$th time step. The choice of $\alpha=1 / 4$ and $\delta=1 / 2$, which corresponds to a constant average-acceleration scheme, is known to give an unconditionally stable scheme in linear problems [35].

Rearranging eqns (48) and (57), we arrive at

$$
\hat{\mathbf{K}} \mathbf{a}^{n+1}=\hat{\mathbf{f}},
$$

where

$$
\begin{aligned}
& \hat{\mathbf{K}}=\mathbf{K}+a_{0} \mathbf{M} \\
& \hat{\mathbf{f}}=\mathbf{f}^{n}+\mathbf{M}\left(a_{0} \mathfrak{a}^{n}+a_{1} \dot{\mathbf{a}}^{n}+a_{2} \ddot{\mathbf{a}}^{n}\right) \\
& a_{0}=1 /\left(\alpha \Delta t^{2}\right) \\
& a_{1}=a_{0} \Delta t \\
& a_{2}=1 /(2 \alpha)-1 .
\end{aligned}
$$

Once the solution $\mathbf{a}$ is known at $t^{n+1}$, the velocity a and acceleration $\mathbf{a}$ at $t^{n+1}$ can be computed by rearranging expressions in eqn (57).

$$
\begin{aligned}
& \ddot{\mathbf{a}}^{n+1}=a_{0}\left(\mathbf{a}^{n+1}-\mathbf{a}^{n}\right)-a_{1} \dot{\mathbf{a}}^{n}-a_{2} \ddot{\mathbf{a}}^{n} \\
& \dot{\mathbf{a}}^{n+1}=\dot{\mathbf{a}}^{n}+a_{3} \ddot{\mathbf{a}}^{n}+a_{4} \ddot{\mathbf{a}}^{n+1},
\end{aligned}
$$

where

$$
a_{3}=\Delta t(1-\delta) \text { and } a_{4}=\delta \Delta t
$$




\subsection{Wilson $-\theta$ method}

Here eqn (48) is first written at time $t^{n+\theta}=t+\theta \Delta t$. If $\tau$ denotes increase in time, where $0 \leqslant \tau \leqslant \theta \Delta t$, then acceleration at time $t+\tau$ is expressed in the form

$$
\ddot{\mathbf{a}}_{t+\tau}=\ddot{\mathbf{a}}_{t}+\tau /(\theta \Delta t)\left[\ddot{\mathbf{u}}_{t+\theta \Delta t}-\ddot{\mathbf{a}}_{t}\right],
$$

where $\theta$ is the parameter that controls the accuracy and stability of the scheme. $\theta \geqslant 1.37$ is known to give an unconditionally stable scheme and usually a value of $\theta=1.4$ is taken.

On integrating twice, the above expression will give

$$
\begin{aligned}
& \dot{\mathbf{a}}_{t+\tau}=\dot{\mathbf{a}}_{t}+\tau \ddot{\mathbf{a}}_{t}+\tau^{2} /(2 \theta \Delta t)\left[\ddot{\mathbf{a}}_{t+\theta \Delta t}-\ddot{\mathbf{a}}_{t}\right] \\
& \mathbf{a}_{t+\tau}=\mathbf{a}_{t}+\tau \dot{\mathbf{a}}_{t}+\tau^{2} / 2 \ddot{\mathbf{a}}_{t}+\tau^{3} /(6 \theta \Delta t)\left[\ddot{\mathbf{a}}_{t+\theta \Delta t}-\ddot{\mathbf{a}}_{t}\right] .
\end{aligned}
$$

Rearranging eqns (48) and (62) will give

$$
\mathbf{R}_{t+0 \Delta t}=\mathbf{f}_{t+\theta \Delta t}
$$

where

$$
\begin{aligned}
\mathbf{R} & =\mathbf{K}+a_{0} \mathbf{M} \\
\mathbf{f}_{t+\theta \Delta t} & =\mathbf{f}_{t}+\theta\left(\mathbf{f}_{t+\Delta t}-\mathbf{f}_{t}\right)+\mathbf{M}\left(a_{0} \mathbf{a}_{t}+a_{1} \dot{\mathbf{a}}_{t}+2 \ddot{\mathbf{a}}_{t}\right) \\
a_{0} & =6 /(\theta \Delta t)^{2} \\
a_{1} & =a_{0} \theta \Delta t .
\end{aligned}
$$

Once the solution a is known at $t+\theta \Delta t$, the acceleration, velocity and displacement at time $t+\Delta t$ can be determined from

$$
\begin{aligned}
& \ddot{\mathbf{a}}_{t+\Delta t}=a_{2}\left(\mathbf{a}_{t+\theta \Delta t}-\mathbf{a}_{t}\right)+a_{3} \dot{\mathbf{a}}_{t}+a_{4} \ddot{\mathbf{a}}_{t} \\
& \dot{\mathbf{a}}_{t+\Delta t}=\dot{\mathbf{a}}_{t}+a_{5}\left(\ddot{\mathbf{a}}_{t+\theta \Delta t}+\ddot{\mathbf{a}}_{t}\right) \\
& \mathbf{a}_{t+\Delta t}=\mathbf{a}_{t}+\Delta t \dot{\mathbf{a}}_{t}+a_{6}\left(\ddot{\mathbf{a}}_{t+v \Delta t}+2 \ddot{\mathbf{a}}_{t}\right),
\end{aligned}
$$

where

$$
\begin{aligned}
& a_{2}=a_{0} / \theta \\
& a_{3}=-a_{1} / \theta \\
& a_{4}=1-3 / \theta \\
& a_{5}=\Delta t / 2 \\
& a_{6}=\Delta t^{2} / 6 .
\end{aligned}
$$

All the operations indicated above, except eqns (60) and (65), can be carried out at the element level and the assembled form of eqns (58) and (63) can be obtained for the whole problem. The equations are then solved for the global solution vector after imposing the boundary conditions of the problem.

\section{NUMERICAL EXAMPLES AND DISCUSSION}

\subsection{Preliminary remarks}

In the present study the nine-noded Lagrangian quadrilateral isoparametric element was employed.
Selective integration scheme, based on Gauss quadrature rules, viz. $3 \times 3$ for membrane, flexure and coupling between membrane and flexure terms, and $2 \times 2$ for shear terms in the energy expression, was employed in the evaluation of the element stiffness property. All the computations were carried in single precision on a CDC Cyber 180/840 computer. Due to the biaxial symmetry of the problems discussed, only quarter plates were analysed using zero initial conditions, i.e. displacement, velocity and acceleration at initial time were considered as zero. All the stress values were reported at the Gauss points nearest to their maximum value locations. For symmetric laminates, the inplane displacements and their higherorder terms were not included in the analysis and only five d.o.f./node displacement model were used to economize on computation time.

The following four sets of data were used in obtaining the numerical results.

Data 1: Square isotropic plate:

$a=25 \mathrm{~cm}, h=5 \mathrm{~cm}$

$E=2.1 \times 10^{6} \mathrm{~N} / \mathrm{cm}^{2}, q_{0}=10 \mathrm{~N} / \mathrm{cm}^{2}$

$\rho=8 \times 10^{-6} \mathrm{~N}-\mathrm{sec}^{2} / \mathrm{cm}^{4}, v=0.25$.

Data 2: Square plate:

$a=25 \mathrm{~cm}, \quad h=5 \mathrm{~cm}, \quad E_{1} / E_{2}=25$

$E_{2}=2.1 \times 10^{6} \mathrm{~N} / \mathrm{cm}^{2}, \quad q_{0}=10 \mathrm{~N} / \mathrm{cm}^{2}$,

$v_{12}=0.25$

$G_{12}=G_{13}=G_{23}=0.5 \mathrm{E}_{2}$,

$\rho=8 \times 10^{-6} \mathrm{~N}-\mathrm{sec}^{2} / \mathrm{cm}^{4}$.

Data 3: Square plate:

$a=140 \mathrm{~mm}, \quad h=4.29 \mathrm{~mm}$

$E_{1}=40 \mathrm{GPa}, \quad E_{z}=8.27 \mathrm{GPa}$,

$\rho=1901.5 \mathrm{~kg} / \mathrm{m}^{3}$

$G_{12}=G_{13}=4.13 \mathrm{GPa}$,

$G_{23}=0.03 \mathrm{GPa}, \quad v_{12}=0.25$.

Data 4: Square plate:

$a=25 \mathrm{~cm}, \quad h=5 \mathrm{~cm}, \quad q_{0}=10 \mathrm{~N} / \mathrm{cm}^{2}$

Face sheets:

$E_{1}=1.308 \times 10^{6} \mathrm{~N} / \mathrm{cm}^{2}, \quad v_{12}=0.28$

$E_{2}=1.06 \times 10^{6} \mathrm{~N} / \mathrm{cm}^{2}$,

$\rho=1.58 \times 10^{-5} \mathrm{~N}-\mathrm{sec}^{2} / \mathrm{cm}^{4}$

$G_{12}=G_{13}=0.6 \times 10^{6} \mathrm{~N} / \mathrm{cm}^{2}$,

$G_{23}=0.39 \times 10^{6} \mathrm{~N} / \mathrm{cm}^{2}$

Core: $\rho=1.009 \times 10^{-6} \mathrm{~N}-\mathrm{sec}^{2} / \mathrm{cm}^{4}$

$G_{13}=5.206 \times 10^{4} \mathrm{~N} / \mathrm{cm}^{2}$,

$G_{23}=1.772 \times 10^{4} \mathrm{~N} / \mathrm{cm}^{2}$. 
Table 1. Estimates of critical time steps $(\mu \mathrm{sec})$

\begin{tabular}{cccccc}
\hline & Data 1 & Data 2 & Data 4 & & Data 3 \\
\hline $1 \times 1$ & 5.86 & 1.17 & 2.31 & Mesh $A$ & 0.425 \\
$2 \times 2$ & 3.42 & 0.68 & 1.65 & Mesh B & 0.120 \\
\hline
\end{tabular}

\subsubsection{Boundary conditions.}

Simply supported:

$$
\begin{aligned}
& \text { along } X \text {-axis } u_{0}=w_{0}=\theta_{x}=u_{0}^{*}=\theta_{x}^{*}=0 \\
& \text { along } Y \text {-axis } v_{0}=w_{0}=\theta_{y}=v_{0}^{*}=\theta_{y}^{*}=0 .
\end{aligned}
$$

Clamped:

all edges

$$
u_{0}=v_{0}=w_{0}=\theta_{x}=\theta_{y}=u_{0}^{*}=v_{0}^{*}=\theta_{x}^{*}=\theta_{y}^{*}=0 .
$$

Along the centre line in the case of quarter plate symmetry:

$$
\begin{aligned}
& \text { along } X \text {-axis } v_{0}=\theta_{y}=v_{0}^{*}=\theta_{y}^{*}=0 \\
& \text { along } Y \text {-axis } u_{0}=\theta_{x}=u_{0}^{*}=\theta_{x}^{*}=0 .
\end{aligned}
$$

In selecting the time step $\Delta t$, the following formula, due to Tsui and Tong, which gives the critical time step for the explicit scheme was used as a guide [36]:

$\Delta t \leqslant\left[\frac{\rho(1-v)^{2}}{E_{1}\left\{2+\pi^{2} / 12\left(1-v^{2}\right)\left[1+1.5(\Delta x / h)^{2}\right]\right\}}\right]^{1 / 2} \cdot \Delta x$,

where $\Delta x$ is the minimum distance between the finite element node points.

\subsection{Examples and discussion}

4.2.1. Example 1. A square simply supported isotropic plate (Data 1) subjected to suddenly applied pulse loading is considered in order to investigate the numerical convergence, the accuracy of the Newmark and Wilson- $\theta$ schemes, the effect of mesh size and the consistent/diagonal mass matrices.

Tables 2 and 3 present maximum centre deflection and maximum centre normal stress for different mesh and time step lengths. Figure 2 shows the convergence of the Newmark and Wilson- $\theta$ schemes; Fig. 3 shows the effect of consistent mass matrix and diagonal mass matrix on transverse deflection.

It can be seen from these tables and plots that, as time step length increases, the Newmark scheme gives better results compared to the Wilson- $\theta$ scheme. It can also be seen that no appreciable different in results is obtained by considering different mass matrices. Figures 4 and 5 show comparison plots of the centre deflection and centre normal stress versus time for the Mindlin plate solution [20] and the HOSD theory solution [29].

4.2.2. Example 2. A two-layer orthotropic crossply $\left[0 / 90^{\circ}\right]$ simply supported square plate (Data 2 ) subjected to a suddenly applied sinusoidally distributed pulse loading given by

$$
q=q_{0} \sin (\pi x / a) \sin (\pi y / a)
$$

is considered. Table 4 presents the maximum centre deflection and maximum stresses for different mesh and time step lengths. Table 5 gives comparison of

\begin{tabular}{|c|c|c|c|c|c|c|c|}
\hline & \multicolumn{3}{|c|}{$1 \times 1$ Mesh } & \multicolumn{3}{|c|}{$2 \times 2$ Mesh } & \multirow{2}{*}{$\frac{3 \times 3 \text { Mesh }}{5}$} \\
\hline$\Delta t(\mu \mathrm{sec})$ & 40 & 10 & 5 & 40 & 10 & 5 & \\
\hline \\
\hline$w\left(\times 10^{3} \mathrm{~cm}\right)$ & $\begin{array}{c}1.5833 \\
(160)\end{array}$ & $\begin{array}{c}1.6818 \\
(160)\end{array}$ & $\begin{array}{c}1.6919 \\
(140)\end{array}$ & $\begin{array}{c}1.5666 \\
(160)\end{array}$ & $\begin{array}{c}1.6721 \\
(160)\end{array}$ & $\begin{array}{c}1.6609 \\
(160)\end{array}$ & $\begin{array}{c}1.6605 \\
(\mathrm{I} 60)\end{array}$ \\
\hline$\sigma_{x}^{t}\left(\mathrm{~N} / \mathrm{cm}^{2}\right)$ & $\begin{array}{c}147.90 \\
(160)\end{array}$ & $\begin{array}{c}158.01 \\
(160)\end{array}$ & $\begin{array}{c}158.86 \\
(140)\end{array}$ & $\begin{array}{c}146.68 \\
(160)\end{array}$ & $\begin{array}{c}161.91 \\
(160)\end{array}$ & $\begin{array}{c}158.83 \\
(160)\end{array}$ & $\begin{array}{c}158.91 \\
(160)\end{array}$ \\
\hline \multicolumn{8}{|l|}{ Consistent mass } \\
\hline \multirow[t]{2}{*}{$w\left(\times 10^{3} \mathrm{~cm}\right)$} & 1.5908 & 1.6751 & 1.6695 & 1.5660 & 1.6758 & - & - \\
\hline & $(160)$ & $(160)$ & $(150)$ & $(160)$ & $(160)$ & - & - \\
\hline \multirow{2}{*}{$\sigma_{x}^{\prime}\left(\mathrm{N} / \mathrm{cm}^{2}\right)$} & 148.59 & 157.09 & 154.89 & 146.91 & 162.68 & - & - \\
\hline & $(160)$ & $(140)$ & $(150)$ & $(160)$ & $(160)$ & - & - \\
\hline
\end{tabular}
stresses at different time steps with closed form solution of Mindlin theory [19].

4.2.3. Example 3. A three-layer orthotropic $\left[0 / 90 / 0^{\circ}\right]$ clamped plate (Data 2 ) subjected to a

Table 2. Convergence of maximum central values for Data 1, Newmark scheme $\dagger$

† Figures in parenthesis indicate time at which maxima occur.

Table 3. Convergence of maximum central values for Data 1 , Wilson- $\theta$ scheme, diagonal mass $\dagger$

\begin{tabular}{lcccccc}
\hline \multicolumn{1}{c}{ Mesh } & \multicolumn{3}{c}{$1 \times 1$} & \multicolumn{3}{c}{$2 \times 2$} \\
\hline$\Delta t(\mu \mathrm{sec})$ & 40 & 10 & 5 & 40 & 10 & 5 \\
$w\left(\times 10^{3} \mathrm{~cm}\right)$ & 1.5092 & 1.6180 & 1.6782 & 1.5782 & 1.6489 & 1.6760 \\
& $(200)$ & $(160)$ & $(140)$ & $(200)$ & $(160)$ & $(160)$ \\
$\sigma_{x}^{t}\left(\mathrm{~N} / \mathrm{cm}^{2}\right)$ & 140.63 & 158.01 & 157.33 & 135.21 & 153.83 & 160.60 \\
& $(200)$ & $(160)$ & $(140)$ & $(200)$ & $(160)$ & $(160)$ \\
\hline
\end{tabular}

† Figures in parenthesis indicate time at which maxima occur. 


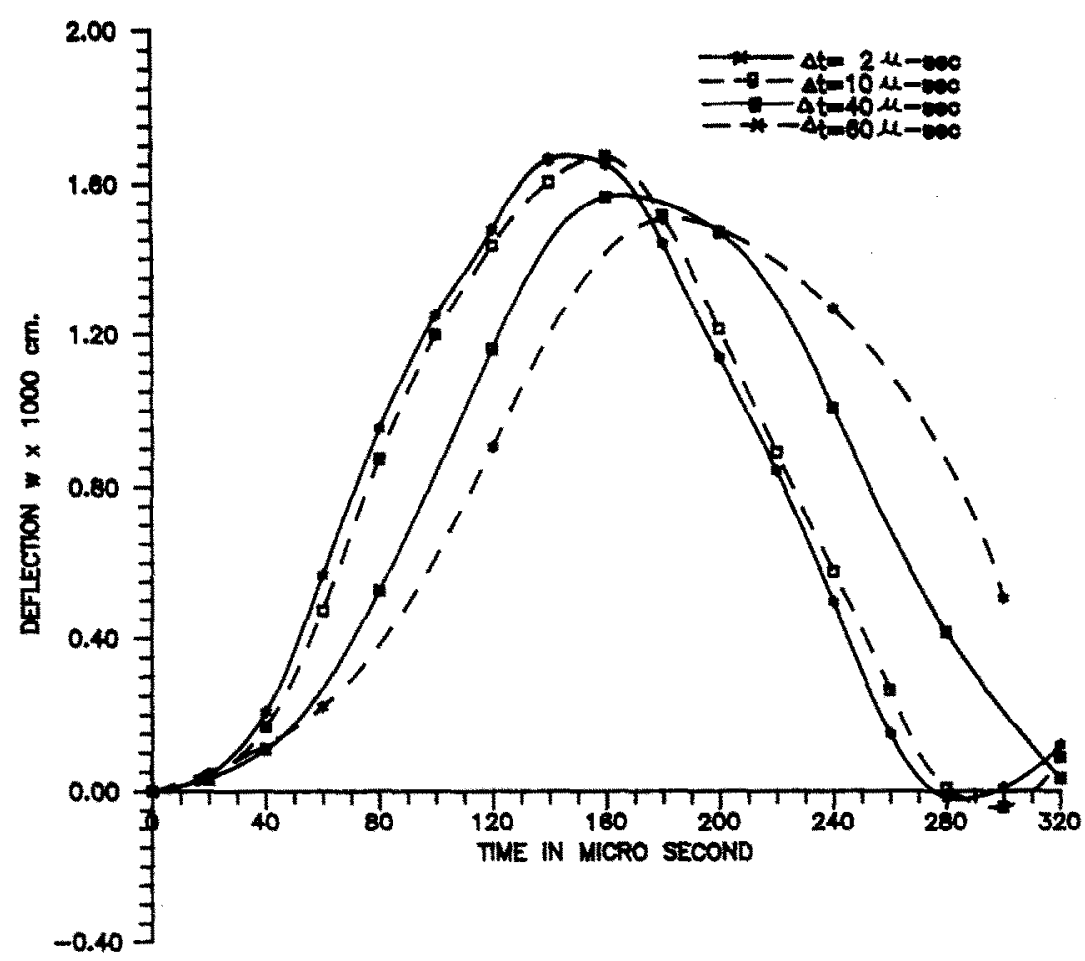

Fig. 2a. Stability of Newmark algorithm for a simply supported isotropic square plate subjected to suddenly applied pulse loading (Data 1, $2 \times 2$ mesh, diagonal mass).

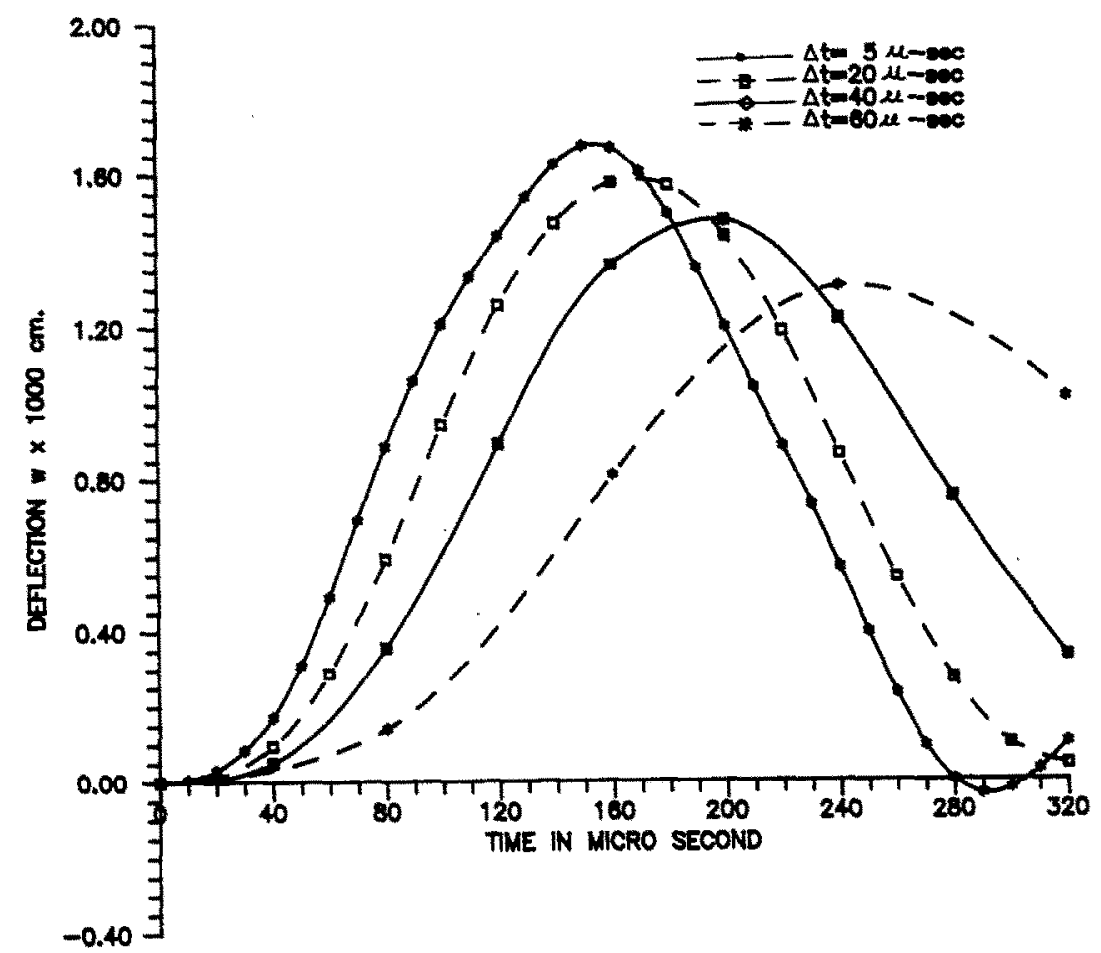

Fig. 2b. Stability of Wilson- $\theta$ algorithm for a simply supported isotropic square plate subjected to suddenly applied pulse loading (Data 1, $2 \times 2$ mesh, diagonal mass).

suddenly applied pulse loading is considered. Figure 6 shows a comparison plot with Mindlin solution [20] and also with HOSD theory solution [29]. It can be seen that Mindlin solution predicts low centre deflec- tion and very low centre normal stress compared to the HOSD theory.

4.2.4. Example 4. A four-layer orthotropic $\left[0 / 90 / 90 / 0^{\circ}\right]$ simply supported plate (Data 2 ) subjected 


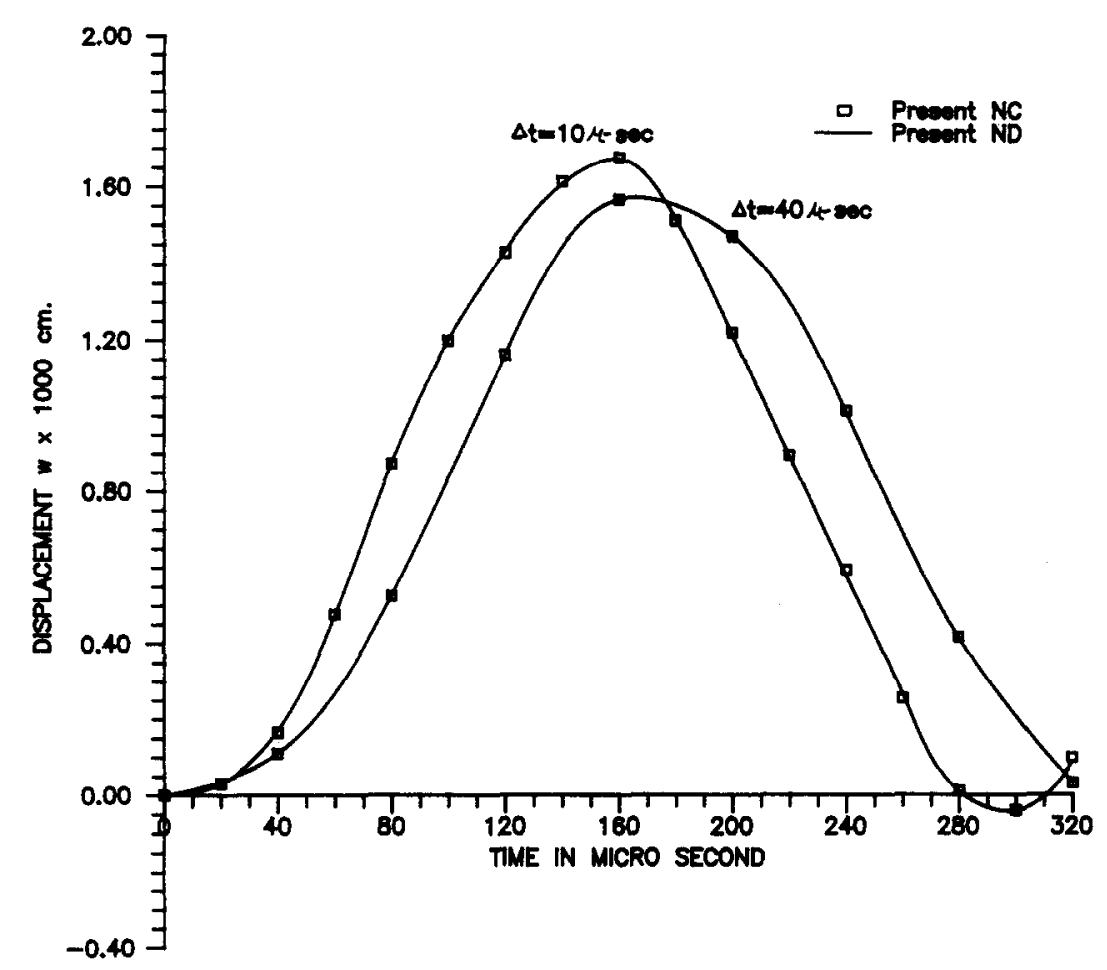

Fig. 3a. Centre deflection versus time for isotropic simply supported square plate subjected to suddenly applied pulse loading (Data 1, $2 \times 2$ mesh).

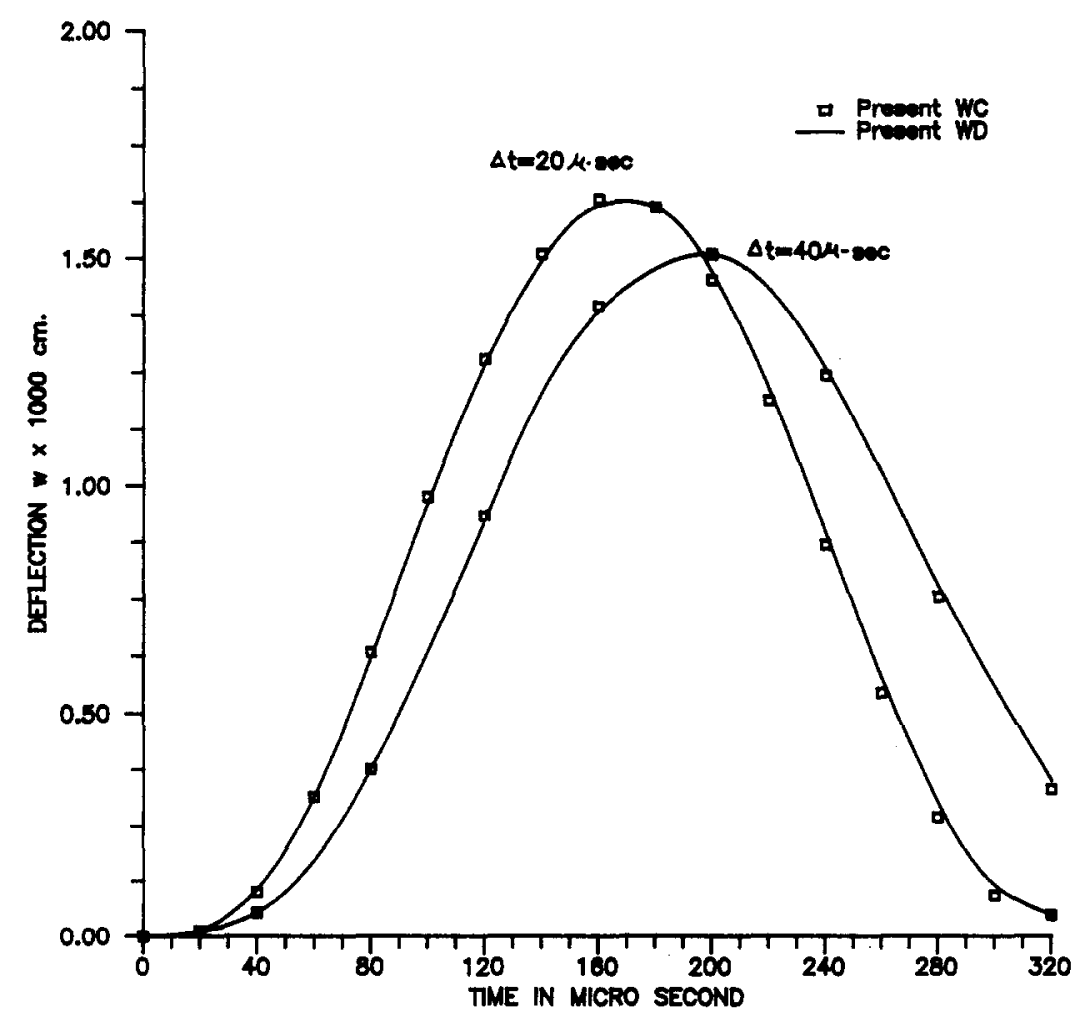

Fig. 3b. Centre deflection versus time for isotropic simply supported square plate subjected to suddenly applied pulse loading (Data $1,1 \times 1$ mesh). 


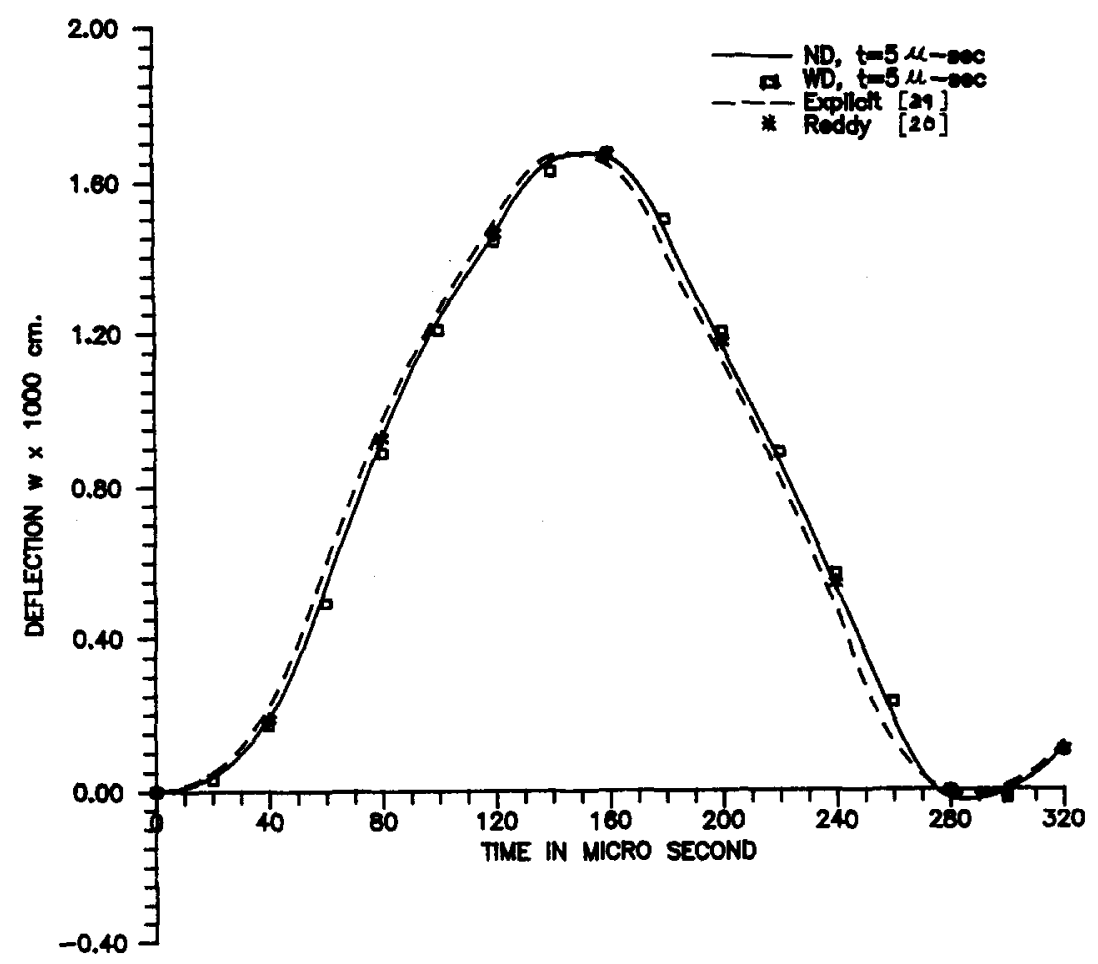

Fig. 4. Centre deflection versus time for isotropic simply supported square plate subjected to suddenly applied pulse loading (Data $1, \mathrm{I} \times 1 \mathrm{mesh}$ ).

to suddenly applied pulse loading is considered. Table 6 gives the maximum centre deflection and maximum centre normal stress for different meshes, time step lengths, and consistent/diagonal mass matrices. Figure 7 shows a comparison plot of centre deflection and centre normal stress with the present HOSD theory using explicit time integration [29]. The effect of consistent and diagonal mass is also shown.

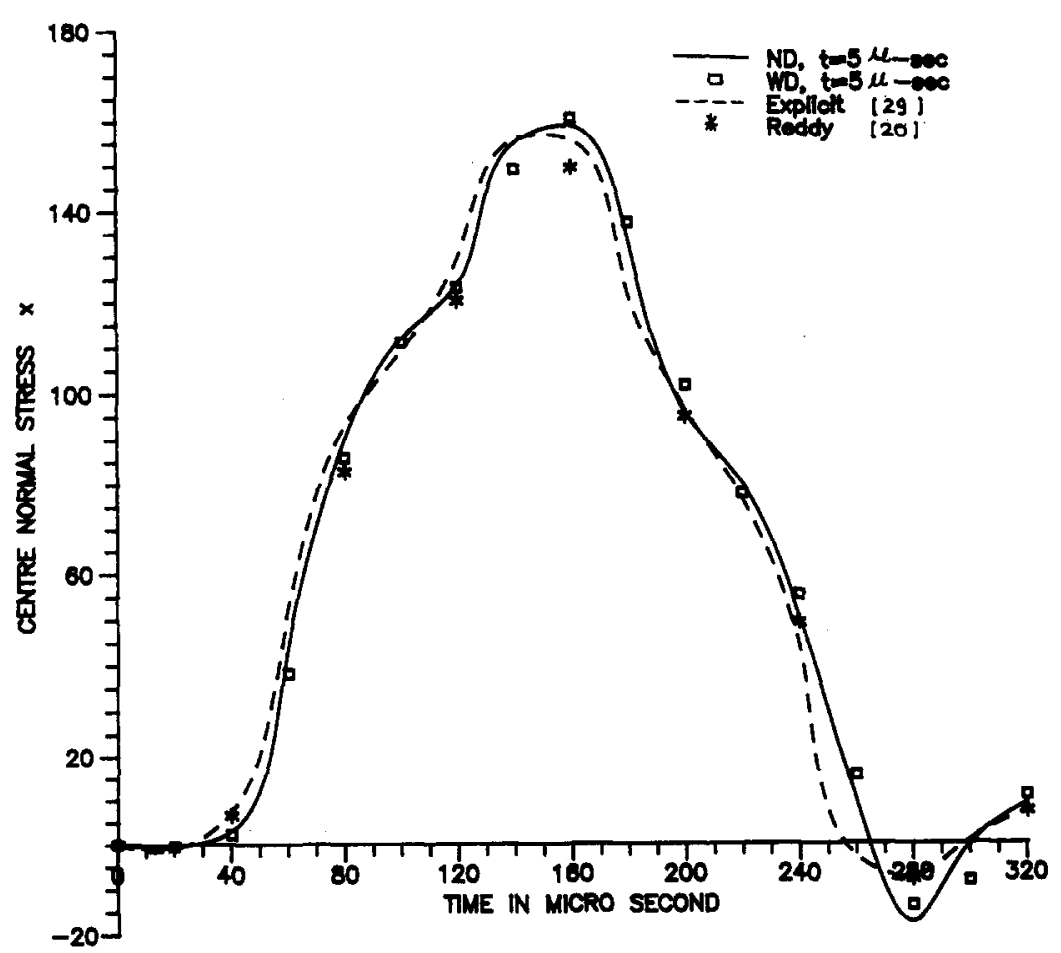

Fig. 5. Centre normal stress versus time for isotropic simply supported square plate subjected to suddenly applied pulse loading (Data 1, $2 \times 2$ mesh). 
Table 4. Convergence of maximum deflection and stresses for two-layer cross-ply $\left(0 / 90^{\circ}\right)$ plate (Data 2 ) under suddenly applied sinusoidal load; Newmark scheme, diagonal mass $†$

\begin{tabular}{|c|c|c|c|c|c|c|c|}
\hline \multicolumn{5}{|c|}{$1 \times 1$ Mesh } & \multicolumn{3}{|c|}{$2 \times 2 \mathrm{Mesh}$} \\
\hline$\Delta t(\mu \mathrm{sec})$ & 40 & 20 & 5 & 2.5 & 40 & 20 & 5 \\
\hline$w\left(\times 10^{3} \mathrm{~cm}\right)$ & $\begin{array}{c}0.4211 \\
(120)\end{array}$ & $\begin{array}{c}0.4541 \\
(120)\end{array}$ & $\begin{array}{c}0.4674 \\
(100)\end{array}$ & $\begin{array}{c}0.4670 \\
(100)\end{array}$ & $\begin{array}{c}0.4235 \\
(120)\end{array}$ & $\begin{array}{c}0.4516 \\
(120)\end{array}$ & $\begin{array}{c}0.4685 \\
(100)\end{array}$ \\
\hline$\sigma_{x}^{\prime}\left(\mathrm{N} / \mathrm{cm}^{2}\right)$ & $\begin{array}{c}320.04 \\
(120)\end{array}$ & $\begin{array}{c}345.06 \\
(120)\end{array}$ & $\begin{array}{c}352.90 \\
(100)\end{array}$ & $\begin{array}{c}355.35 \\
(100)\end{array}$ & $\begin{array}{c}321.45 \\
(120)\end{array}$ & $\begin{array}{c}343.24 \\
(120)\end{array}$ & $\begin{array}{c}355.26 \\
(100)\end{array}$ \\
\hline$\tau_{x=}\left(\mathrm{N} / \mathrm{cm}^{2}\right)$ & $\begin{array}{c}17.804 \\
(120)\end{array}$ & $\begin{array}{c}19.083 \\
(120)\end{array}$ & $\begin{array}{c}19.845 \\
(110)\end{array}$ & $\begin{array}{c}19.960 \\
(100)\end{array}$ & $\begin{array}{c}19.608 \\
(120)\end{array}$ & $\begin{array}{c}21.117 \\
(120)\end{array}$ & $\begin{array}{c}21.720 \\
(100)\end{array}$ \\
\hline$\tau_{x y}\left(\mathrm{~N} / \mathrm{cm}^{2}\right)$ & $\begin{array}{c}26.465 \\
(120)\end{array}$ & $\begin{array}{c}28.054 \\
(120)\end{array}$ & - & $\begin{array}{c}29.133 \\
(100)\end{array}$ & $\begin{array}{c}26.472 \\
(120)\end{array}$ & $\begin{array}{c}28.175 \\
(120)\end{array}$ & $\begin{array}{c}29.280 \\
(100)\end{array}$ \\
\hline
\end{tabular}

$\dagger$ Figures in parenthesis indicate time at which maxima occur.

Table 5. Comparision of stresses obtained in the present study with those by Reissner-Mindlin plate theory for two-layer $\left(0 / 90^{\circ}\right)$ simply-supported plate (Data 2$)$ under suddenly applied sinusoidal load $\dagger$

\begin{tabular}{crcrrrr}
\hline$t$ & \multicolumn{2}{c}{$\sigma_{x}\left(\mathrm{~N} / \mathrm{cm}^{2}\right)$} & \multicolumn{2}{c}{$\tau_{x y}\left(\mathrm{~N} / \mathrm{cm}^{2}\right)$} & \multicolumn{2}{c}{$\tau_{x z}\left(\mathrm{~N} / \mathrm{cm}^{2}\right)$} \\
$(\mu \mathrm{sec})$ & Present & CFS [19] & Present & CFS [19] & Present & CFS [19] \\
\hline 20 & 30.03 & 28.48 & 1.937 & 1.611 & 2.547 & 2.252 \\
40 & 111.68 & 113.60 & 9.228 & 8.506 & 6.991 & 5.891 \\
60 & 224.41 & 227.20 & 18.190 & 16.470 & 14.067 & 12.340 \\
80 & 316.78 & 319.10 & 26.050 & 23.850 & 19.450 & 16.340 \\
100 & 355.26 & 357.80 & 29.280 & 26.270 & 21.720 & 18.940 \\
120 & 326.87 & 323.10 & 26.646 & 24.120 & 20.295 & 15.960 \\
140 & 237.08 & 233.00 & 19.710 & 17.050 & 14.408 & 12.580 \\
160 & 130.17 & 119.60 & 10.128 & 8.848 & 8.583 & 6.533 \\
180 & 35.39 & 30.40 & 2.976 & 2.029 & 2.222 & 2.233 \\
200 & 3.94 & 0.742 & 0.304 & 0.248 & -1.027 & 0.564 \\
\hline
\end{tabular}

† Newmark scheme; diagonal mass; $2 \times 2$ mesh; $\Delta t=5 \mu \mathrm{sec}$.

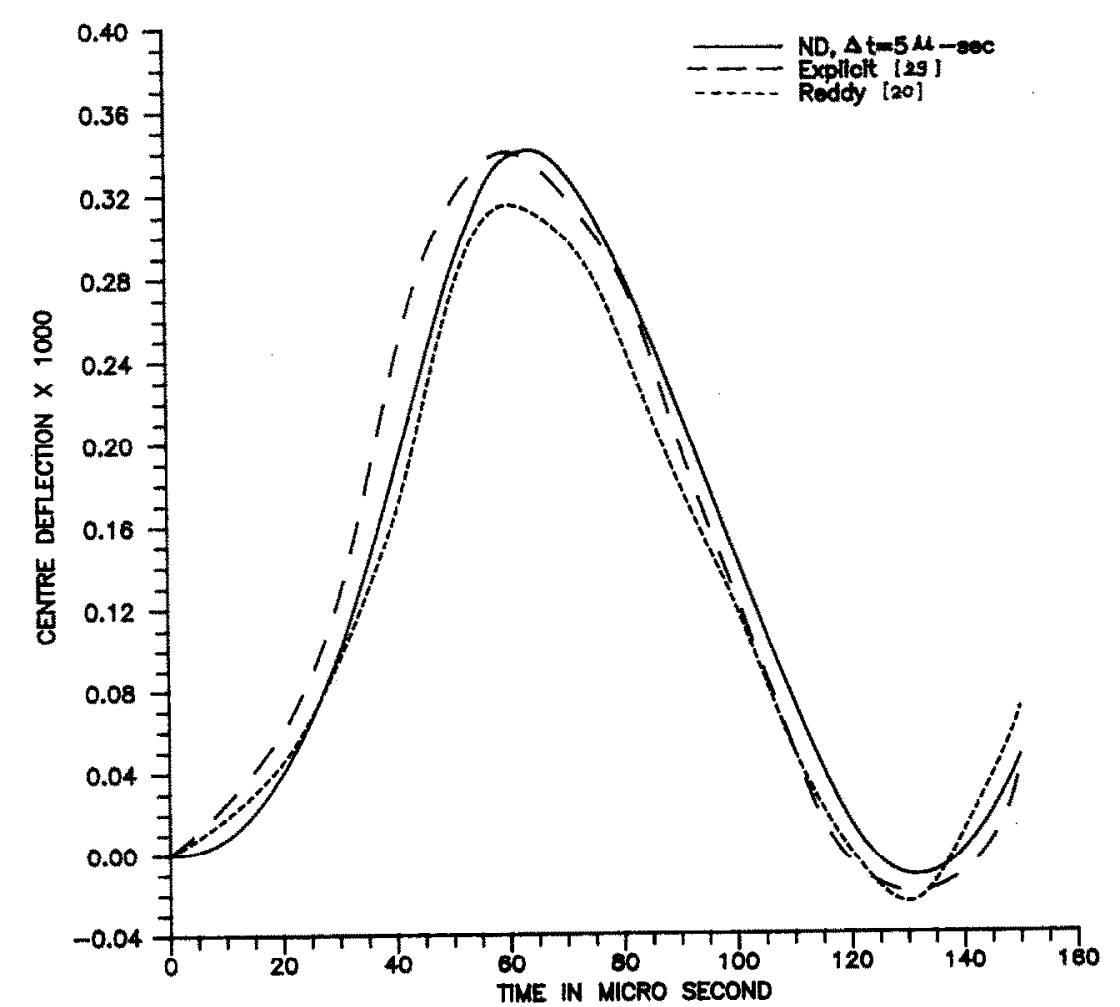

Fig. 6a. Centre deflection versus time for three-layered $\left(0 / 90 / 0^{\circ}\right)$ simply supported square plate subjected to suddenly applied pulse loading (Data $2,2 \times 2$ mesh). 


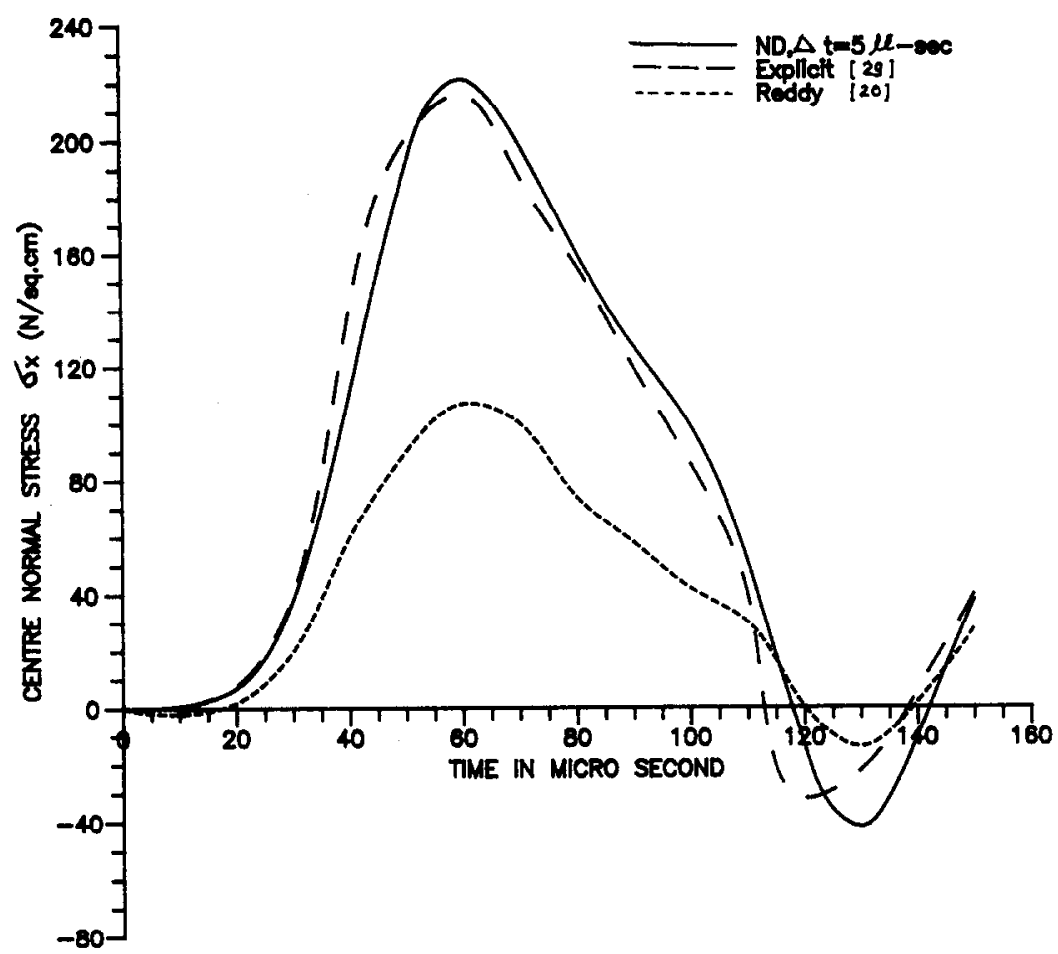

Fig. 6b. Centre normal stress versus time for three-layered $\left(0 / 90 / 0^{\circ}\right)$ clamped square plate subjected to suddenly applied pulse loading (Data $2,2 \times 2$ mesh).

4.2.5. Example 5. A three-layer graphite/epoxy $\left[0 / 90 / 0^{\circ}\right]$ clamped plate (Data 3 ) subjected to central impact loading is of a small cylindrically shaped blunt-ended projectile is considered. The blunt-ended impactor has the following characteristics:

$$
\text { diameter }=9.525 \mathrm{~mm} ; \quad \text { mass }=0.01417 \mathrm{~kg} \text {. }
$$

The interaction force between the impactor and the laminate is assumed to be uniformly distributed over the contact area and is calculated by the Hertz law of contact, which is written as [37]

$$
F=H(r-w)^{p}
$$

where $F$ is the impactor-plate interaction force, $H$ is a constant which depends upon the plate material and is chosen to be equal to $10^{8} \mathrm{~N} / \mathrm{m}^{1.5}, r$ is the distance travelled by the impactor, $w$ is the displacement of the centre of the plate; and $p$ is an exponent which depends upon the geometry of the impactor and is set equal to 1.5 [38]. Figure 8 shows the finite element mesh considered for the analysis.

Figure 9a shows a comparison of centre transverse deflection with experimental results as well as finite element results [38] using the theory of Yang et al. [5]. Figures $9 \mathrm{~b}$ and $\mathrm{c}$ give centre normal stress $\sigma_{x}^{\text {top }}$ and applied distributed load as a function of time. The advantage of the Newmark scheme is quite evident in this problem. From Fig. $9 \mathrm{~b}$ it can be seen that the Newmark scheme gives accurate results for $\Delta t=25 \mu \mathrm{sec}$, which is approximately 200 times $\Delta t_{\text {cr }}$ of the explicit scheme.

4.2.6. Example 6. A $\left[0 / 90 /\right.$ core $\left./ 90 / 0^{\circ}\right]$ simply supported sandwich plate (Data 4) subjected to suddenly applied pulse loading is analysed to consider the effect

Table 6. Convergence of maximum central values for Data 2, Newmark scheme, $0 / 90 / 90 / 0^{\circ}$ plate†

\begin{tabular}{lcccccc}
\hline & \multicolumn{3}{c}{$1 \times 1$ Mesh } & \multicolumn{3}{c}{$2 \times 2$ Mesh } \\
\hline$\Delta t(\mu \mathrm{sec})$ & 40 & 10 & 5 & 40 & 10 & 5 \\
Diagonal mass & & & & & & \\
$w\left(\times 10^{3} \mathrm{~cm}\right)$ & 0.4369 & 0.4793 & 0.4806 & 0.4312 & 0.4806 & 0.4773 \\
& $(120)$ & $(80)$ & $(80)$ & $(120)$ & $(80)$ & $(80)$ \\
$\sigma_{x}^{t}\left(\mathrm{~N} / \mathrm{cm}^{2}\right)$ & 413.41 & 454.76 & 458.75 & 397.46 & 433.18 & 426.74 \\
& $(120)$ & $(80)$ & $(80)$ & $(120)$ & $(80)$ & $(80)$ \\
Consistent mass & & & & & & \\
$w\left(\times 10^{3} \mathrm{~cm}\right)$ & 0.4404 & 0.4806 & 0.4907 & 0.4321 & 0.4770 & 0.4756 \\
& $(120)$ & $(80)$ & $(80)$ & $(120)$ & $(80)$ & $(80)$ \\
$\sigma_{x}^{t}\left(\mathrm{~N} / \mathrm{cm}^{2}\right)$ & 415.44 & 454.92 & 468.61 & 398.76 & 429.35 & 422.20 \\
& $(120)$ & $(80)$ & $(80)$ & $(120)$ & $(80)$ & $(80)$ \\
\hline
\end{tabular}

\footnotetext{
$\dagger$ Figures in parenthesis indicate time at which maxima occur.
} 


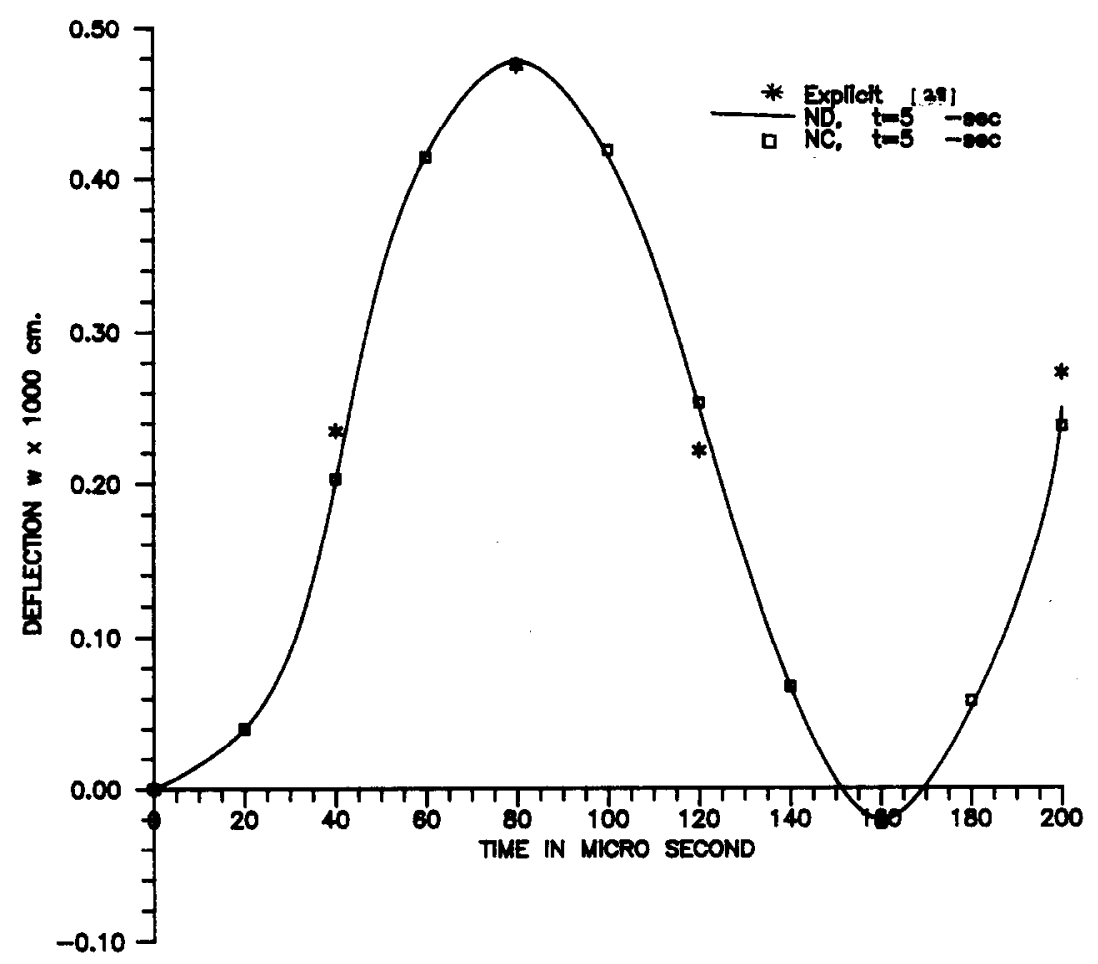

Fig. 7a. Centre deflection versus time for four-layered $\left(0 / 90 / 90 / 0^{\circ}\right)$ simply supported square plate subjected to suddenly applied pulse loading (Data $2,2 \times 2$ mesh).

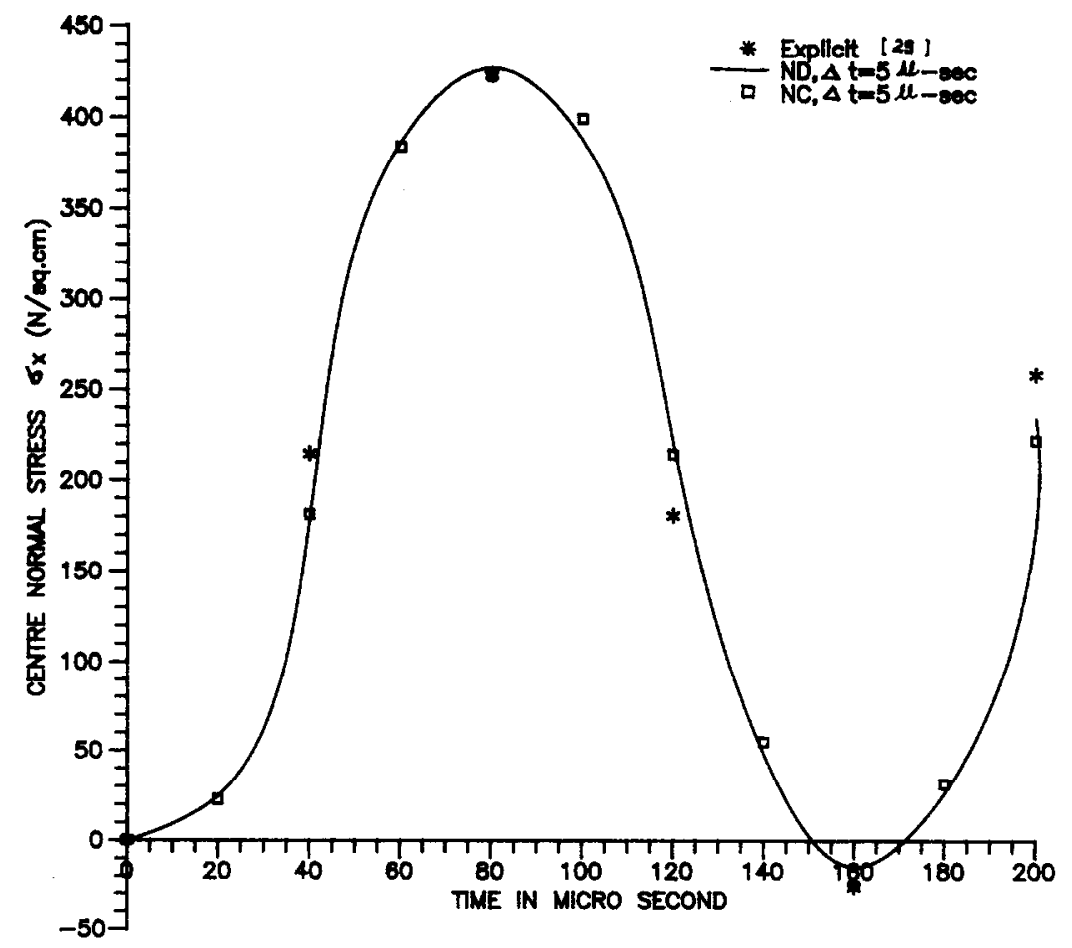

Fig. $7 \mathrm{~b}$. Centre normal stress versus time for four-layered $\left(0 / 90 / 90 / 0^{\circ}\right)$ simply supported square plate subjected to suddenly applied pulse loading (Data $2,2 \times 2$ mesh). 


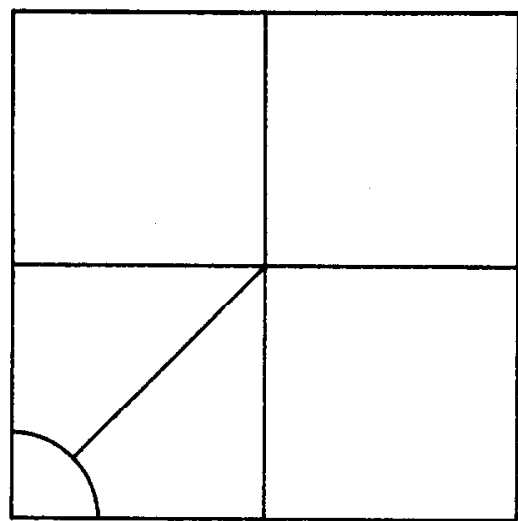

Mesh-A

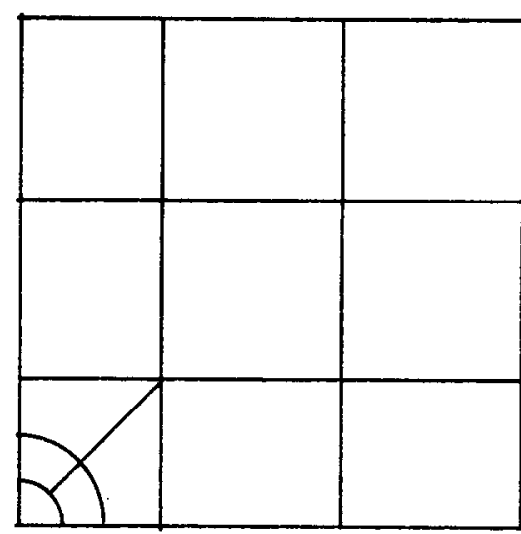

Mesh-B

Fig. 8. Finite element mesh for projectile problem.

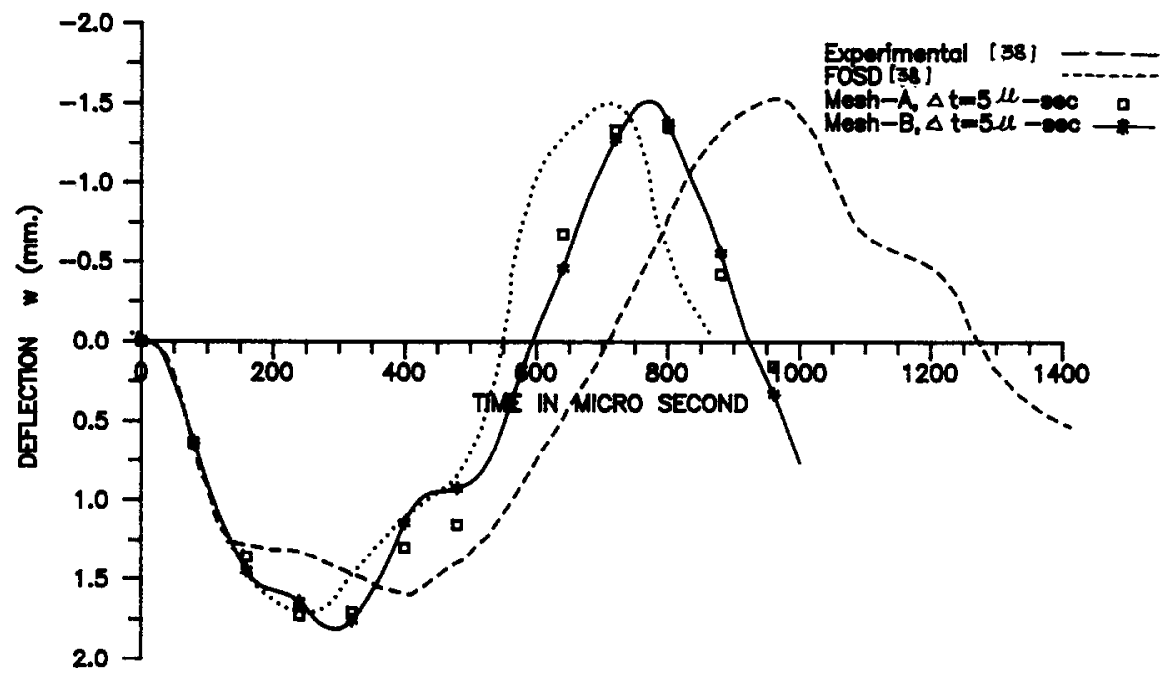

Fig. 9a. Centre deflection versus time for three-layered $\left(0 / 90 / 0^{\circ}\right)$ clamped square plate (Data 3 ).

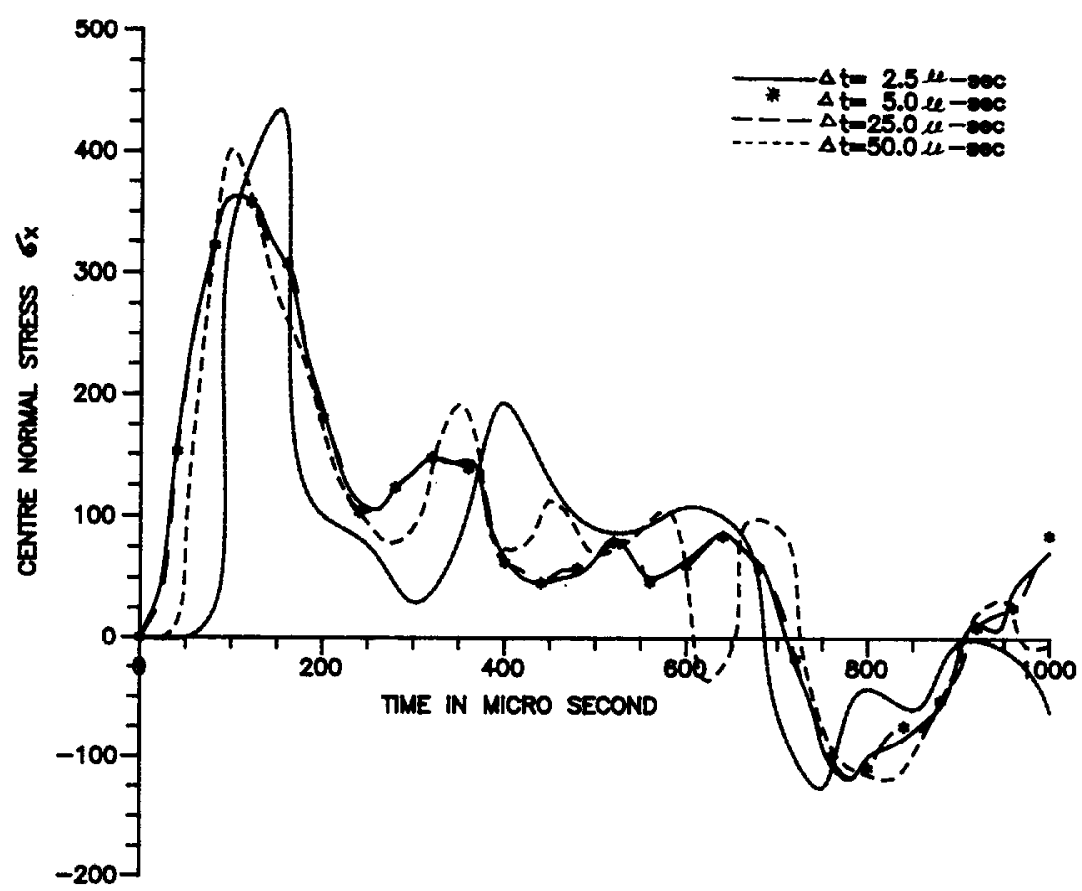

Fig. 9b. Centre normal stress versus time for three-layered $\left(0 / 90 / 0^{\circ}\right)$ clamped square plate (Data 3 ). 


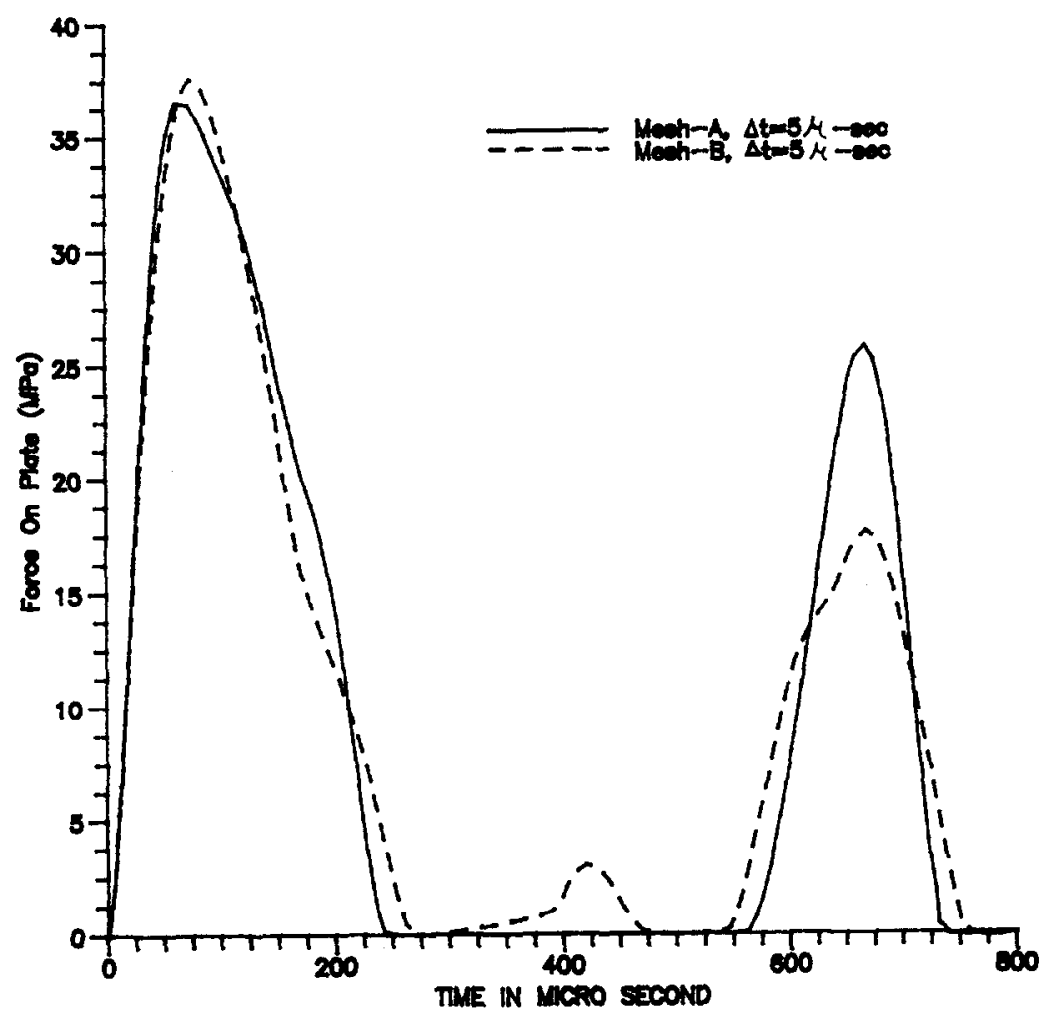

Fig. 9c. Applied force as a function of time for a three-layered laminate $\left(0 / 90 / 0^{\circ}\right)(h=4.29 \mathrm{~mm}$, $v=22.6 \mathrm{~m} / \mathrm{sec}, a=140 \mathrm{~mm}$, Data 3 ).

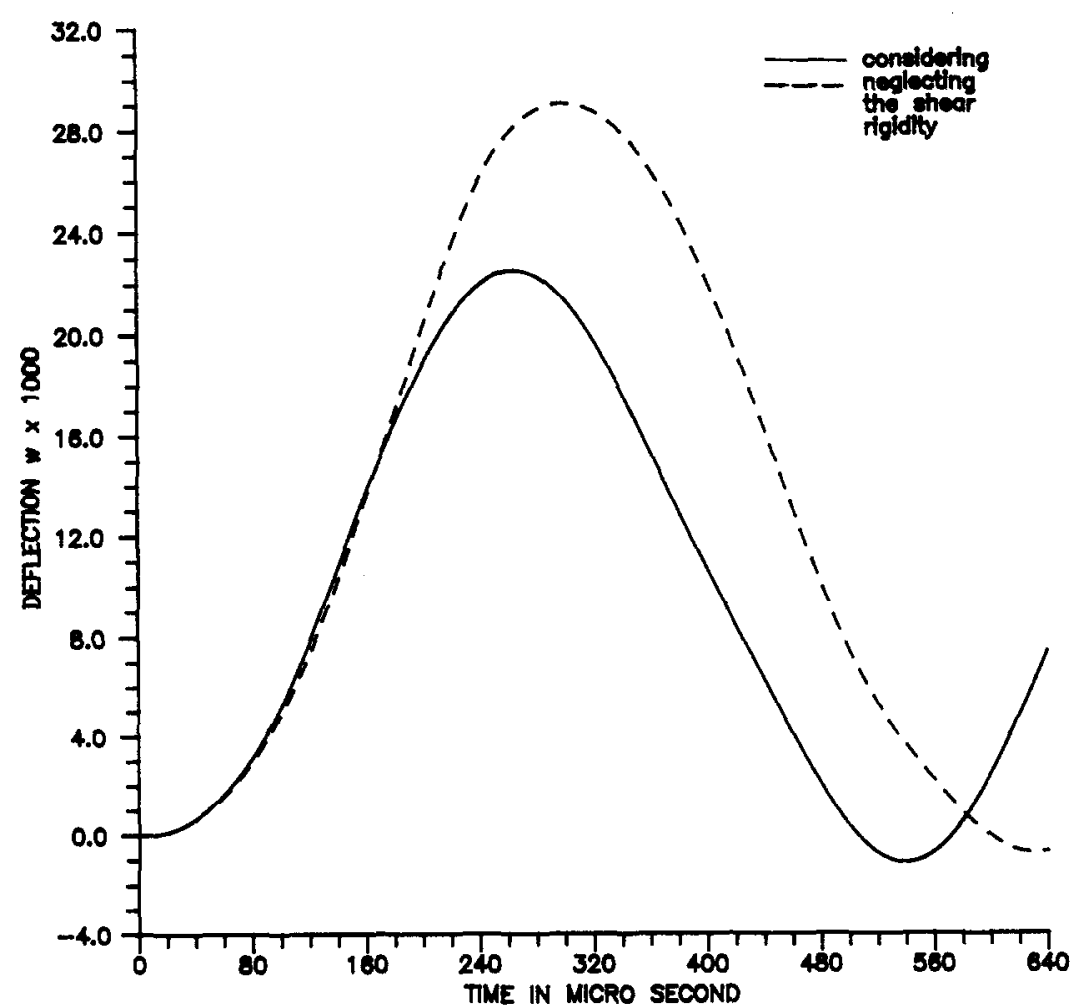

Fig. 10a. Centre deflection versus time for a simply supported square sandwich $\left(0 / 90 /\right.$ core $\left./ 90 / 0^{\circ}\right)$ plate under suddenly applied pulse loading (Data $4,2 \times 2$ mesh, Newmark scheme, diagonal mass, $t=10 \mu \mathrm{sec}$ ). 


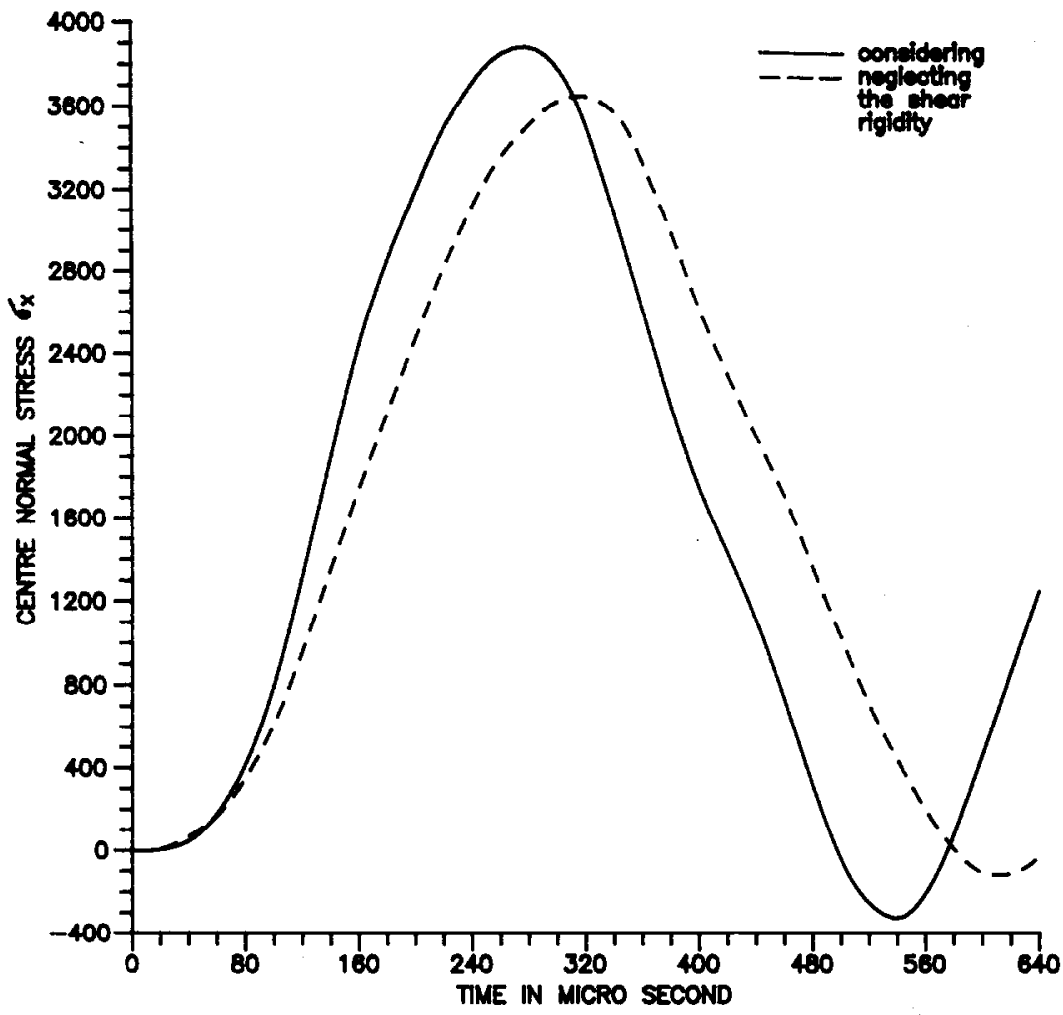

Fig. 10b. Centre normal stress versus time for a simply supported square sandwich $\left(0 / 90 /\right.$ core $\left./ 90 / 0^{\circ}\right)$ plate under suddenly applied pulse loading (Data 4, $2 \times 2$ mesh, Newmark scheme, diagonal mass, $t=10 \mu \mathrm{sec}$ ).

of shear rigidities of the stiff layers on the dynamic response.

Figure 10 shows maximum centre deflection and maximum centre normal stress $\sigma_{x}^{t}$. It can be seen that the effect of neglecting shear rigidities of stiff layers is to overestimate the maximum centre deflection by approximately $25-30 \%$, whereas there is no appreciable difference in the centre normal stress.

\section{CONCLUSIONS}

A higher order shear deformable theory with $\mathrm{C}^{0}$ plate element is employed for the transient dynamic analysis of composite plates. Through the comparative study done here, we clearly see the importance of this theory for highly anisotropic plates. In contrast to the classical shear deformation theory, the present theory does not require a shear correction coefficient due to the more realistic representation of crosssectional deformation.

The advantages in the use of the higher order theory presented here over the Reissner/Mindlin theory is not quite evident for isotropic plates, but such usage is shown to be very effective in the analysis of nonhomogeneous, anisotropic, composite or sandwich systems, as the mathematical model on which this theory is based is far superior to the Mindlin theory.

Also, it has been shown that consistent and diagonal mass matrices give identical results. For larger time step lengths the Newmark scheme is found to be more accurate than the Wilson- $\theta$ scheme. The advantage of using an implicit scheme is shown by taking a time step of the order of 10-200 times the critical time step length of the explicit scheme, depending upon the problem, and by obtaining quite accurate results, having an error of $\pm 10 \%$.

Acknowledgements-Partial support of this research by the Aeronautics Research and Development Board, Ministry of Defence, Government of India through its Grants Aero/RD-134/100/84-85/362, Aero/RD-134/100/10/88$89 / 518$ and Aero/RD-134/100/10/88-89/534 is gratefully acknowledged.

\section{REFERENCES}

1. E. Reissner and Y. Stavsky, Bending and stretching of certain types of heterogeneous aelotropic elastic plates. J. appl. Mech., ASME 28, 402-408 (1961).

2. S. B. Dong, K. S. Pister and R. L. Taylor, On the theory of laminated anisotropic shells and plates. J. Aerospace Sci. 29, 269-275 (1962).

3. E. Reissner, The effect of transverse shear deformation on the bending of elastic plates. J. appl. Mech., ASME 12, A69-A77 (1945).

4. R. D. Mindlin, Influence of rotary inertia and shear on flexural motions of isotropic elastic plates. J. appl. Mech., ASME 18, 31-38 (1951).

5. P. C. Yang, C. H. Norris and Y. Stavsky, Elastic wave propagation in heterogeneous plates. Int. J. Solids Struct. 2, 665-684 (1966).

6. J. M. Whitney and N. J. Pagano, Shear deformation in heterogeneous anisotropic plates. J. appl. Mech., ASME 37, 1031-1036 (1970).

7. H. Reismann, Forced motion of elastic plates. J. appl. Mech., ASME 35, 510-515 (1968). 
8. H. Reismann and $Y$. Lee, Forced motion of rectangular plates. In Developments in Theoretical and Applied Mechanics (Edited by D. Fredrick), Vol. 4, pp. 3-18. Pergamon, New York (1969).

9. Y. Lee and H. Reismann, Dynamics of rectangular plates. Int. J. Engng Sci. 7, 93-113 (1969).

10. T. Rock and E. Hinton, Free vibration and transient response of thick and thin plates using the finite element method. Earthquake Engng Struct. Dynam. 3, 51-63 (1974)

11. T. S. Chow, On the propagation of flexural waves in an orthotropic laminated plate and its response to an impulsive load. J. Comp. Mater. 5, 306-319 (1971).

12. A. S. D. Wang, P. C. Chow and J. L. Rose, Strongly coupled stress waves in heterogeneous plates. Am. Inst. Aeronaut. Astronaut. Jnl 10, 1088-1090 (1972).

13. F. C. Moon, Wave surface due to impact on anisotropic plates. J. Comp. Mater. 6, 62-69 (1972).

14. F. C. Moon, One dimensional transient waves in anisotropic plates. J. appl. Mech. 40, 485-490 (1973).

15. C. T. Sun and J. M. Whitney, Forced vibrations of laminated composite plates in cylindrical bending. J. acous. Soc. Am. 55, 1003-1008 (1974).

16. C. T. Sun and J. M. Whitney, Dynamic response of laminated composite plates. Am. Inst. Aeronaut. Astronaut. Jnl 13, 1259-1260 (1975).

17. J. M. Whitney and C. T. Sun, Transient response of laminated composite plates subjected to transverse dynamic loading. J. acoust. Soc. Am. 61, 101-104 (1975).

18. R. D. Mindlin and L. F. Goodman, Beam vibrations with time-dependent boundary conditions. $J$, appl. Mech. 17, 377-380 (1950).

19. J. N. Reddy, On the solutions to forced motions of rectangular composite plates. J. appl. Mech. 49, 403-408 (1982).

20. J. N. Reddy, Dynamic (transient) analysis of layered anisotropic composite-material plates. Int. J. Numer. Meth. Engng 19, 237-255 (1983).

21. K. H. Lo, R, M. Christensen and E. M. Wu, A higher order theory of plate deformation-part 2: laminated plates. J. appl. Mech. 44, 669-676 (1977).

22. T. Kant, Numerical analysis of thick plates. Comput. Meth. appl. Mech. Engng 31, 1-18 (1982).

23. N. D. Phan and J. N. Reddy, Analysis of laminated composite plates using a higher-order shear deformation theory. Int. J. Numer. Meth. Engng 21, 2201-2219 (1985).
24. T. Kant, D. R. J. Owen and O. C. Zienkiewicz, A refined higher-order $\mathrm{C}^{0}$ plate bending element. Comput. Struct. 15, 177-183 (1982).

25. B. N. Pandya and T. Kant, A consistent refined theory for flexure of a symmetric laminate. Mech. Res. Commun. 14, 107-113 (1987).

26. B. N. Pandya and T. Kant, A refined higher-order generally orthotropic $\mathrm{C}^{0}$ plate bending element. Comput. Struct. 29, 119-133 (1987).

27. B. N. Pandya and T. Kant, Flexural analysis of laminated composite using refined higher-order $\mathrm{C}^{0}$ plate bending element. Comput. Meth. appl. Mech. Engng 66, 173-198 (1988).

28. B. N. Pandya and T. Kant, Finite element analysis of laminated composite plate using a higher-order displacement model. Comp. Sci. Technol. 32, 137-155 (1988).

29. Mallikarjuna and T. Kant, Dynamics of laminated composite plates with a higher-order theory and finite element discretization. $J$. Sound Vibr. 126, 463-475 (1988).

30. T. Kant, R. V. Ravichandran, B. N. Pandya and Mallikarjuna, Finite element analysis of isotropic and fibre reinforced composite plates using a higher-order theory. Comp. Struct. 9, 319-342 (1988).

31. R. M. Jones, Mechanics of Composite Materials. McGraw-Hill, New York (1975).

32. E. Hinton, T. A. Rock and O. C. Zienkiewicz, A note on mass lumping and related processes in finite element methods. Int. J. Earthquake Engng struct. Dynam. 4, 245-249 (1976).

33. R. D. Cook, Concepts and Applications of Finite Element Analysis. John Wiley, New York (1981)

34. K. J. Bathe, Finite Element Procedures in Engineering Analysis. Prentice-Hall, Englewood Cliffs, NJ (1982).

35. T. J. R. Hughes, The Finite Element Method: Linear Static and Dynamic Finite Element Analysis. PrenticeHall, Englewood Cliffs, NJ (1987).

36. T. Y. Tsui and P. Tong, Stability of transient solution of moderately thick plates by finite difference methods. Am. Inst. Aeronaut. Astronaut. Jnl 3, 1772-1773 (1971).

37. W. Goldsmith, Impact, The Theory and Physical Behaviour of Colliding Bodies. Edward Arnold, London (1960).

38. H. Aggour and C. T. Sun, Finite element analysis of laminated composite plate subjected to circularly distributed impact loading. Comput. Struct. 28, 729-736 (1988). 